\title{
Scalar modes of the relic gravitons
}

\author{
Massimo Giovannini* \\ Department of Physics, Theory Division, CERN, 1211 Geneva 23, Switzerland INFN, \\ Section of Milan-Bicocca, 20126 Milan, Italy \\ (Received 20 October 2014; published 29 January 2015)
}

\begin{abstract}
In conformally flat background geometries the long-wavelength gravitons can be described in the fluid approximation and they induce scalar fluctuations both during inflation and in the subsequent radiationdominated epoch. While this effect is minute and suppressed for a de Sitter stage of expansion, the fluctuations of the energy-momentum pseudotensor of the graviton fluid lead to curvature perturbations that increase with time all along the post-inflationary evolution. An explicit calculation of these effects is presented for a standard thermal history and it is shown that the growth of the curvature perturbations caused by the long-wavelength modes is approximately compensated by the slope of the power spectra of the energy density, pressure and anisotropic stress of the relic gravitons.
\end{abstract}

DOI: 10.1103/PhysRevD.91.023521

PACS numbers: 98.80.Cq, 04.30.-w, 04.62.+v, 98.70.Vc

\section{INTRODUCTION}

The tensor modes of the geometry can be efficiently amplified in conformally flat space-times thanks to the pumping action of the (extrinsic) curvature [1]. As a consequence, relic gravitons are often regarded as a direct signature of any scenario positing an early variation of the background gravitational field: typical examples are the conventional inflationary paradigm [2-4] and various completions of the concordance lore $[5,6]$.

The energy and momentum of the gravitational field itself cannot be localized. This perspective is vividly described, for instance, in Ref. [7] (see also Ref. [8]) and it rests on the validity of the equivalence principle. Since there is no unique expression for the energymomentum tensor of the relic gravitons, we must instead deal with a variety of pseudotensors whose definitions are mathematically different but physically equivalent. The classic Landau and Lifshitz approach [9] stipulates that a valid energy-momentum pseudotensor can be obtained (in Minkowski space) from the second-order fluctuations of the Einstein tensor (see also Refs. [10,11]). The same strategy pioneered in a Minkowski background can be appropriately extended to conformally flat space-times [12]. In a complementary perspective Ford and Parker [13] suggested that the second-order action for the tensor modes of the geometry in a conformally flat space-time of Friedmann-Robertson-Walker type coincides with the action of two minimally coupled scalar fields (one for each tensor polarization): it is then plausible to argue that the energy-momentum tensor of each polarization of the

*massimo.giovannini@cern.ch

Published by the American Physical Society under the terms of the Creative Commons Attribution 3.0 License. Further distribution of this work must maintain attribution to the author(s) and the published article's title, journal citation, and DOI. graviton must coincide with that of a minimally coupled scalar field.

In the present investigation it is observed that the fluctuations of the energy density, of the pressure and of the anisotropic stress of the relic gravitons produce secondary scalar modes of the geometry. During inflation their contribution can be safely ignored: while the energy density of the inflaton is approximately constant the energy density of the gravitons is suppressed. Conversely, during the postinflationary phase the contribution of the long-wavelength gravitons to the curvature perturbations increases both during radiation and during the matter-dominated epoch. The rationale of this effect is, in short, the following. The fluctuations of the energy-momentum pseudotensor decrease more slowly than the dominant component of the energy density, at least in the case of the conventional thermal history dominated by radiation and, later on, by dust. To gauge the robustness of the physical results, the analysis will be conducted within the two complementary parametrizations of the energy-momentum pseudotensor mentioned in the previous paragraph.

The relic graviton fluid and its fluctuations are introduced in Sec. II. In Sec. III we examine the evolution of the scalar modes induced by an effective fluid of relic gravitons. Section IV contains the evaluation of the various power spectra and the explicit estimates of the curvature perturbations. The concluding remarks are collected in Sec. V. To avoid digressions, some technical results of the analysis have been collected in the Appendices.

\section{THE FLUID OF RELIC GRAVITONS}

\section{A. Generalities}

While the energy and momentum of the gravitational field itself cannot be localized, there exist consistent frameworks for the analysis of a gravitational energymomentum pseudotensor on a given background geometry 
(be it flat space-time or any other space-time). In the present situation we shall be chiefly concerned with conformally flat geometries. ${ }^{1}$ Let us first consider the case where the energy-momentum of the sources (denoted hereafter by $T_{\mu \nu}$ ) vanishes; the Einstein equations can then be written in the form ${ }^{2}$

$$
\delta^{(1)} \mathcal{G}_{\mu}^{\nu}=\ell_{\mathrm{P}}^{2} \mathcal{U}_{\mu}^{\nu},
$$

where $\mathcal{G}_{\mu}^{\nu}=R_{\mu}^{\nu}-\delta_{\mu}^{\nu} R / 2$ is the Einstein tensor written in terms of the Ricci tensor and of the Ricci scalar denoted, respectively, by $R_{\mu}^{\nu}$ and $R$. In Eq. (2.1) $\delta^{(1)} \mathcal{G}_{\mu}^{\nu}$ stands as a symbol for the first-order fluctuation of the Einstein tensor. By definition $\mathcal{U}_{\mu}^{\nu}$ is the energy-momentum tensor of the gravitational field itself and it depends on the first-order fluctuations of the Einstein tensor:

$$
\mathcal{U}_{\mu}^{\nu}=\frac{1}{\ell_{\mathrm{P}}^{2}}\left[\delta^{(1)} \mathcal{G}_{\mu}^{\nu}-\mathcal{G}_{\mu}^{\nu}\right]
$$

The explicit form of $\mathcal{U}_{\mu}^{\nu}$ depends on the transformation properties of the fluctuations with respect to three-dimensional rotations and also on the specific background geometry. In general terms the first-order fluctuations of the Einstein tensor can be written as

$$
\delta^{(1)} \mathcal{G}_{\mu}^{\nu}=\delta_{\mathrm{t}}^{(1)} \mathcal{G}_{\mu}^{\nu}+\delta_{\mathrm{v}}^{(1)} \mathcal{G}_{\mu}^{\nu}+\delta_{\mathrm{s}}^{(1)} \mathcal{G}_{\mu}^{\nu},
$$

where the subscripts on the right-hand side denote, respectively the tensor, vector and scalar fluctuations of $\mathcal{G}_{\mu}^{\nu}$.

For a conventional post-inflationary thermal history the vector modes can be neglected since they are always suppressed both during and after inflation. ${ }^{3}$ Thus, using Eq. (2.3), Eqs. (2.1) and (2.2) can be written as ${ }^{4}$

$$
\begin{gathered}
\delta_{\mathrm{t}}^{(1)} \mathcal{G}_{\mu}^{\nu}=0, \\
\delta_{\mathrm{s}}^{(1)} \mathcal{G}_{\mu}^{\nu}=\ell_{\mathrm{P}}^{2}\left[\delta_{\mathrm{s}}^{(1)} T_{\mu}^{\nu}+\mathcal{U}_{\mu}^{\nu}\right], \\
\mathcal{U}_{\mu}^{\nu}=-\frac{1}{\ell_{\mathrm{P}}^{2}} \delta_{\mathrm{t}}^{(2)} \mathcal{G}_{\mu}^{\nu},
\end{gathered}
$$

\footnotetext{
${ }^{1}$ The metric tensor of the background metric is given, in the present context, by $\bar{g}_{\mu \nu}=a^{2}(\tau) \eta_{\mu \nu} ; a(\tau)$ is the scale factor, $\tau$ denotes the conformal time coordinate and $\eta_{\mu \nu}$ is the Minkowski metric with signature $(+,-,-,-)$.

${ }^{2}$ The Planck length will be defined as $\ell_{\mathrm{P}}=\sqrt{8 \pi G}$ in units $\hbar=c=1$.

${ }^{3}$ In the present investigation the attention shall be focused on the concordance lore with standard post-inflationary thermal history.

${ }^{4}$ Note that $T_{\mu}^{\nu}$ is the energy-momentum tensor of the matter field that has now been included for a consistent treatment of the scalar modes of the geometry.
}

where the fluctuations of $T_{\mu}^{\nu}$ have been included by assuming, as in the conventional case, that $\delta_{\mathrm{t}}^{(1)} T_{\mu}^{\nu}=$ $\delta_{\mathrm{v}}^{(1)} T_{\mu}^{\nu}=0$. The superscript on the right-hand side of Eq. (2.6) denotes the second-order fluctuation of the corresponding quantity while the subscript refers to the tensor nature of the fluctuations. The components of the energy-momentum pseudotensor $\mathcal{U}_{\mu}^{\nu}$ are not covariantly conserved. However, since the Bianchi identity $\nabla_{\mu} \mathcal{G}_{\nu}^{\mu}=0$ should be valid to all orders, we will also have to demand $\delta_{\mathrm{t}}^{(2)}\left(\nabla_{\mu} \mathcal{G}_{\nu}^{\mu}\right)=0$. The perturbation of the Bianchi identity implies that the relic graviton fluid, in the Landau-Lifshitz parametrization, possesses an effective bulk viscosity (see Appendix B for a discussion of this point). Equation (2.6) defines the Landau-Lisfshitz pseudotensor in its general form. Moreover, Eqs. (2.4), (2.5), and (2.6) concisely summarize all the logical steps of the present discussion. In particular, the explicit form of Eq. (2.4) gives the standard evolution of the tensor fluctuations of the geometry while Eq. (2.5) formally accounts for the evolution of the scalar modes modified by the relic gravitons whose pseudotensor is given by Eq. (2.6).

A complementary strategy for a suitable definition of the energy-momentum pseudotensor of the relic gravitons has been suggested in Ref. [13]. In a conformally flat background $\bar{g}_{\mu \nu}=a^{2}(\tau) \eta_{\mu \nu}$, the Einstein-Hilbert action perturbed to second order in the amplitude of the tensor modes, reads, up to total derivatives,

$$
S_{\mathrm{gw}}=\delta_{\mathrm{t}}^{(2)} S=\frac{1}{8 \ell_{\mathrm{P}}} \int d^{4} x \sqrt{-\bar{g}} \bar{g}^{\alpha \beta} \partial_{\alpha} h_{i j} \partial_{\beta} h_{i j},
$$

where $\bar{g}_{\mu \nu}(\tau)=a^{2}(\tau) \eta_{\mu \nu}$ and the first-order fluctuation of the metric is defined as $g_{\mu \nu}(\vec{x}, \tau) \rightarrow \bar{g}_{\mu \nu}(\tau)+\delta_{\mathrm{t}}^{(1)} g_{\mu \nu}(\vec{x}, \tau)$. Furthermore $\delta_{\mathrm{t}}^{(1)} g_{0 i}(\vec{x}, \tau)=\delta_{\mathrm{t}}^{(1)} g_{00}(\vec{x}, \tau)=0$ and

$$
\delta_{\mathrm{t}}^{(1)} g_{i j}(\vec{x}, \tau)=-a^{2}(\tau) h_{i j}(\vec{x}, \tau), \quad \partial_{i} h_{j}^{i}=h_{i}^{i}=0 .
$$

The energy-momentum pseudotensor is formally obtained from Eq. (2.7) by functional derivation with respect to $\bar{g}_{\mu \nu}$, i.e.

$$
\delta S=\frac{1}{2} \int d^{4} x \sqrt{-\bar{g}} \mathcal{W}_{\mu \nu} \delta \bar{g}_{\mu \nu}
$$

As expected, by setting to zero the variation of Eq. (2.7) with respect to $h_{i j}$, we obtain the evolution of the amplitude of the tensor fluctuations in our conformally flat background geometry:

$$
h_{i j}^{\prime \prime}+2 \mathcal{H} h_{i j}^{\prime}-\nabla^{2} h_{i j}=0,
$$

where the prime denotes a derivation with respect to the conformal time coordinate $\tau$ and, as usual, $\mathcal{H}=a^{\prime} / a$. 
Equation (2.10) has the same content as Eq. (2.4) since $\delta_{\mathrm{t}}^{(1)} R=0$ and $\delta_{\mathrm{t}}^{(1)} \mathcal{G}_{0}^{0}=\delta_{\mathrm{t}}^{(1)} \mathcal{G}_{i}^{0}=0$.

\section{B. Explicit forms of the pseudotensors}

According to Eq. (2.2) the energy-momentum pseudotensor can be computed from the second-order fluctuations of the Einstein tensor after a straightforward but algebraically lengthy procedure. Some of the relevant results of this calculation were reported in the first paper of Ref. [12] and subsequently rederived (see second paper of Ref. [12] and references therein). The main result for the components of the energy-momentum pseudotensor can be expressed, in the conformal time parametrization, as follows:

$\mathcal{U}_{0}^{0}=\frac{1}{a^{2} \ell_{\mathrm{P}}^{2}}\left[\mathcal{H} H_{k \ell} h^{k \ell}+\frac{1}{8}\left(\partial_{m} h_{k \ell} \partial^{m} h^{k \ell}+H_{k \ell} H^{k \ell}\right)\right]$,

$\mathcal{U}_{i}^{j}=\frac{\mathcal{U}}{3} \delta_{i}^{j}+\Pi_{i}^{j}, \quad \mathcal{U}_{i}^{0}=\frac{1}{4 \ell_{\mathrm{P}}^{2} a^{2}} H_{k \ell} \partial_{i} h_{k \ell}$,

where

$$
\begin{aligned}
\mathcal{U}= & \frac{1}{8 a^{2} \ell_{\mathrm{P}}^{2}}\left[5 H_{k \ell} H^{k \ell}-7 \partial_{m} h_{k \ell} \partial^{m} h^{k \ell}\right], \\
\Pi_{i}^{j}= & \frac{1}{a^{2} \ell_{\mathrm{P}}^{2}}\left\{\frac{1}{6}\left[H_{k \ell} H^{k \ell}-\frac{1}{2} \partial_{m} h_{k \ell} \partial^{m} h^{k \ell}\right] \delta_{i}^{j}\right. \\
& \left.+\frac{1}{2} \partial_{m} h_{\ell i} \partial^{m} h^{\ell j}-\frac{1}{4} \partial_{i} h_{k \ell} \partial^{j} h^{k \ell}-\frac{1}{2} H_{k i} H^{k j}\right\},
\end{aligned}
$$

with $\Pi_{i}^{i}=0$. In Eqs. (2.11)-(2.12) and (2.13)-(2.14) we used the notation

$$
\partial_{\tau} h_{i j}=h_{i j}^{\prime}=H_{i j}, \quad \mathcal{H}=\frac{a^{\prime}}{a} .
$$

In a complementary perspective, from Eq. (2.9), the energy-momentum pseudotensor can be expressed as

$$
\mathcal{W}_{\mu}^{\nu}=\frac{1}{4 \ell_{\mathrm{P}}^{2} a^{2}}\left[\partial_{\mu} h_{i j} \partial^{\nu} h^{i j}-\frac{1}{2} \delta_{\mu}^{\nu} \bar{g}^{\alpha \beta} \partial_{\alpha} h_{i j} \partial_{\beta} h^{i j}\right],
$$

or, in components,

$\mathcal{W}_{0}^{0}=\frac{1}{8 \ell_{\mathrm{P}}^{2} a^{2}}\left[H_{k \ell} H^{k \ell}+\partial_{m} h_{k \ell} \partial_{m} h^{k \ell}\right]$

$\mathcal{W}_{i}^{j}=\frac{\mathcal{W}}{3} \delta_{i}^{j}+\Pi_{i}^{j}, \quad \mathcal{W}_{i}^{0}=\frac{1}{4 \ell_{\mathrm{P}}^{2} a^{2}} H_{k \ell} \partial_{i} h^{k \ell}$,

where $\Pi_{i}^{i}=0$ and, moreover,

$$
\begin{aligned}
& \mathcal{W}=\frac{1}{8 \ell_{\mathrm{P}}^{2} a^{2}}\left[\partial_{m} h_{k \ell} \partial_{m} h^{k \ell}-3 H_{k \ell} h^{k \ell}\right], \\
& \Pi_{i}^{j}=\frac{1}{4 \ell_{\mathrm{P}}^{2} a^{2}}\left[-\partial_{i} h_{k \ell} \partial^{j} h^{k \ell}+\frac{1}{3} \delta_{i}^{j} \partial_{m} h_{k \ell} \partial_{m} h_{k \ell}\right] .
\end{aligned}
$$

\section{Polarizations of the tensor modes}

With the aim of discussing more explicit expressions of $\mathcal{U}_{\mu}^{\nu}$ and $\mathcal{W}_{\mu}^{\nu}$ it is useful to introduce the two polarizations of the gravitons:

$$
e_{i j}^{(\oplus)}(\hat{k})=\left(\hat{m}_{i} \hat{m}_{j}-\hat{n}_{i} \hat{n}_{j}\right), \quad e_{i j}^{(\otimes)}(\hat{k})=\left(\hat{m}_{i} \hat{n}_{j}+\hat{n}_{i} \hat{m}_{j}\right),
$$

where $\hat{k}$ is oriented along the direction of propagation of the wave while $\hat{m}_{i}=m_{i} /|\vec{m}|$ and $\hat{n}=n_{i} /|\vec{n}|$ are mutually orthogonal and orthogonal to $\hat{k}$. It follows from Eq. (2.21) that $e_{i j}^{(\lambda)} e_{i j}^{\left(\lambda^{\prime}\right)}=2 \delta_{\lambda \lambda^{\prime}}$ while the sum over the polarizations gives

$$
\begin{aligned}
\sum_{\lambda} e_{i j}^{(\lambda)}(\hat{k}) e_{m n}^{(\lambda)}(\hat{k})= & {\left[p_{m i}(\hat{k}) p_{n j}(\hat{k})+p_{m j}(\hat{k}) p_{n i}(\hat{k})\right.} \\
& \left.-p_{i j}(\hat{k}) p_{m n}(\hat{k})\right]
\end{aligned}
$$

with $p_{i j}(\hat{k})=\left(\delta_{i j}-\hat{k}_{i} \hat{k}_{j}\right)$. The Fourier transform of $h_{i j}(\vec{x}, \tau)$ is defined as

$$
h_{i j}(\vec{k}, \tau)=\frac{1}{(2 \pi)^{3 / 2}} \sum_{\lambda} \int d^{3} k h_{i j}(\vec{x}, \tau) e^{i \vec{k} \cdot \vec{x}} .
$$

In the quantum description of the evolution of the gravitons $h_{i j}(\vec{x}, \tau)$ and $H_{i j}(\vec{x}, \tau)$ are interpreted as field operators in the Heisenberg description, namely

$$
\begin{aligned}
\hat{h}_{i j}(\vec{x}, \tau)= & \frac{\sqrt{2} \ell_{\mathrm{P}}}{(2 \pi)^{3 / 2} a} \sum_{\lambda} \int d^{3} k e_{i j}^{(\lambda)}(\vec{k})\left[f_{k, \lambda}(\tau) \hat{a}_{\vec{k} \lambda}\right. \\
& \left.\times e^{-i \vec{k} \cdot \vec{x}}+f_{k, \lambda}^{*}(\tau) \hat{a}_{\vec{k} \lambda}^{\dagger} e^{i \vec{k} \cdot \vec{x}}\right],
\end{aligned}
$$

$$
\begin{aligned}
\hat{H}_{i j}(\vec{x}, \tau)= & \frac{\sqrt{2} \ell_{\mathrm{P}}}{(2 \pi)^{3 / 2} a} \sum_{\lambda} \int d^{3} k e_{i j}^{(\lambda)}(\vec{k})\left[g_{k, \lambda}(\tau) \hat{a}_{\vec{k} \lambda}\right. \\
& \left.\times e^{-i \vec{k} \cdot \vec{x}}+g_{k, \lambda}^{*}(\tau) \hat{a}_{\vec{k} \lambda}^{\dagger} e^{i \vec{k} \cdot \vec{x}}\right],
\end{aligned}
$$

where $f_{k}$ and $g_{k}=\left(f_{k}^{\prime}-\mathcal{H} f_{k}\right)$ are the corresponding mode functions; the creation and annihilation operators obey, as usual, $\left[\hat{a}_{\vec{k}, \lambda}, \hat{a}_{\vec{k}^{\prime}, \lambda^{\prime}}^{\dagger}\right]=\delta_{\lambda \lambda^{\prime}} \delta^{(3)}\left(\vec{k}-\vec{k}^{\prime}\right)$. In the parametrization (2.23) $\hat{h}_{i j}(\vec{k}, \tau)$ and $\hat{H}_{i j}(\vec{k}, \tau)$ are given by: 


$$
\begin{aligned}
\hat{h}_{i j}(\vec{k}, \tau)= & \frac{\sqrt{2} \ell_{\mathrm{P}}}{a} \sum_{\lambda}\left[e_{i j}^{(\lambda)}(\vec{k}) f_{k, \lambda}(\tau) \hat{a}_{\vec{k} \lambda}\right. \\
& \left.+e_{i j}^{(\lambda)}(-\vec{k}) f_{k, \lambda}^{*}(\tau) \hat{a}_{-\vec{k} \lambda}\right], \\
\hat{H}_{i j}(\vec{k}, \tau)= & \frac{\sqrt{2} \ell_{\mathrm{P}}}{a} \sum_{\lambda}\left[e_{i j}^{(\lambda)}(\vec{k}) g_{k, \lambda}(\tau) \hat{a}_{\vec{k} \lambda}\right. \\
& \left.+e_{i j}^{(\lambda)}(-\vec{k}) g_{k, \lambda}^{*}(\tau) \hat{a}_{-\vec{k} \lambda}\right] .
\end{aligned}
$$

From Eq. (2.26) the two-point functions of the operators can be expressed in terms of the related power spectra:

$$
\begin{aligned}
\left\langle\hat{h}_{i j}(\vec{k}, \tau) \hat{h}_{m n}\left(\vec{k}^{\prime}, \tau\right)\right\rangle & =\frac{2 \pi^{2}}{k^{3}} P_{f f}(k, \tau) \delta^{(3)}\left(\vec{k}+\vec{k}^{\prime}\right) \mathcal{S}_{i j m n}(\hat{k}), \\
\left\langle\hat{H}_{i j}(\vec{k}, \tau) \hat{H}_{m n}\left(\vec{k}^{\prime}, \tau\right)\right\rangle & =\frac{2 \pi^{2}}{k^{3}} P_{g g}(k, \tau) \delta^{(3)}\left(\vec{k}+\vec{k}^{\prime}\right) \mathcal{S}_{i j m n}(\hat{k}), \\
\left\langle\hat{h}_{i j}(\vec{k}, \tau) \hat{H}_{m n}\left(\vec{k}^{\prime}, \tau\right)\right\rangle & =\frac{2 \pi^{2}}{k^{3}} P_{f g}(k, \tau) \delta^{(3)}\left(\vec{k}+\vec{k}^{\prime}\right) \mathcal{S}_{i j m n}(\hat{k}), \\
\left\langle\hat{H}_{i j}(\vec{k}, \tau) \hat{h}_{m n}\left(\vec{k}^{\prime}, \tau\right)\right\rangle & =\frac{2 \pi^{2}}{k^{3}} P_{g f}(k, \tau) \delta^{(3)}\left(\vec{k}+\vec{k}^{\prime}\right) \mathcal{S}_{i j m n}(\hat{k}) .
\end{aligned}
$$

The power spectra appearing in Eq. (2.27) are expressible in terms of the corresponding mode functions:

$$
\begin{gathered}
P_{f f}(k, \tau)=\frac{4 \ell_{\mathrm{P}}^{2} k^{3}}{\pi^{2} a^{2}(\tau)}\left|f_{k}(\tau)\right|^{2}, \\
P_{g g}(k, \tau)=\frac{4 \ell_{\mathrm{P}}^{2} k^{3}}{\pi^{2} a^{2}(\tau)}\left|g_{k}(\tau)\right|^{2}, \\
P_{f g}(k, \tau)=\frac{4 \ell_{\mathrm{P}}^{2} k^{3}}{\pi^{2} a^{2}(\tau)} f_{k}(\tau) g_{k}^{*}(\tau), \\
P_{g f}(k, \tau)=\frac{4 \ell_{\mathrm{P}}^{2} k^{3}}{\pi^{2} a^{2}(\tau)} f_{k}^{*}(\tau) g_{k}(\tau) .
\end{gathered}
$$

In Eq. (2.27) we introduced the following quantity:

$$
\mathcal{S}_{i j m n}(\hat{k})=\frac{1}{4} \sum_{\lambda} e_{i j}^{(\lambda)}(\hat{k}) e_{m n}^{(\lambda)}(\hat{k})
$$

Recalling Eqs. (2.21) and (2.22), it is easy to derive the following identity:

$$
\begin{aligned}
\mathcal{Q}(\vec{a}, \vec{b}) & =\mathcal{S}_{i j m n}(\hat{a}) \mathcal{S}_{i j m n}(\hat{b}) \\
& =\frac{\left[1+(\hat{a} \cdot \hat{b})^{2}\right]\left[1+3(\hat{a} \cdot \hat{b})^{2}\right]}{16},
\end{aligned}
$$

where $\hat{a}=\vec{a} /|\vec{a}|$ and $\hat{b}=\vec{b} /|\vec{b}|$. If $\hat{a}$ and $\hat{b}$ coincide $\mathcal{Q}(\hat{a}, \hat{a}) \rightarrow 1 / 2$.

\section{THE SCALAR MODES OF THE RELIC GRAVITON FLUID}

\section{A. Governing equations}

In the long-wavelength limit (i.e. when $k \tau \ll 1$ ) the modes of the field are amplified and the fluctuations of the pressure and of the energy density depend on the salient features of the background evolution. In the opposite regime, i.e. $k \tau \gg 1$ (short-wavelength limit) the Fourier modes of the tensor fluctuations of the geometry oscillate. From the discussion of Sec. IV the perturbed entries are always the same but their relation with the amplitudes of the two tensor polarizations depends on the specific parametrization (be it $\mathcal{W}_{\mu}^{\nu}$ or $\mathcal{U}_{\mu}^{\nu}$ ). For the purposes of the present section it is sufficient to posit the following general form ${ }^{5}$ for the components of $\delta_{\mathrm{s}} \mathcal{T}_{\mu}^{\nu}$ :

$\delta_{\mathrm{s}} \mathcal{T}_{0}^{0}=\delta \rho_{\mathrm{gw}}, \quad \delta_{\mathrm{s}} \mathcal{T}_{i}^{0}=\mathcal{J}_{i}=\frac{H_{k \ell} \partial_{i} h^{k \ell}}{4 \ell_{\mathrm{P}}^{2} a^{2}}$,

$\delta_{\mathrm{s}} \mathcal{T}_{i}^{j}=-\delta p_{\mathrm{gw}} \delta_{i}^{j}+\Pi_{i}^{j}$.

The fluctuations of the energy density and pressure are

$\rho_{\mathrm{gw}}(\vec{x}, \tau)=\left\langle\rho_{\mathrm{gw}}\right\rangle+\delta \rho_{\mathrm{gw}}, \quad p_{\mathrm{gw}}(\vec{x}, \tau)=\left\langle p_{\mathrm{gw}}\right\rangle+\delta p_{\mathrm{gw}}$,

where $\langle\ldots .$.$\rangle denotes the expectation value on the quantum$ state of the relic gravitons ${ }^{6}$; the expectation values can also be viewed as stochastic averages by using the correspondence between the power spectra and the mode functions outlined in Eq. (2.27). Different forms of pseudotensors [see e.g. Eqs. (2.6) and (2.16)] modify the actual expressions of $\delta \rho_{\mathrm{gw}}, \delta p_{\mathrm{gw}}$ and $\Pi_{i}^{j}$. In Sec. IV this point will be examined in detail but the relation between the induced curvature perturbations and Eq. (3.1) is independent of the specific parametrization.

In the sudden reheating approximation, the quasi-de Sitter stage of expansion lasts up to a fiducial time $\tau_{1}$ marking simultaneously the end of inflation and the onset of radiation. In the short-wavelength regime we have a collection of relativistic bosons; thus the pressure fluctuations will be given by $\delta p_{\mathrm{gw}}=\delta \rho_{\mathrm{gw}} / 3$. This implies that the short-wavelength fluctuations of the energy density and of the pressure are exponentially suppressed during the de Sitter stage of expansion while the background energy density is nearly constant.

\footnotetext{
${ }^{5}$ Recalling the considerations of Sec. II, $\mathcal{T}_{\mu}^{\nu}$ may either coincide with $\mathcal{U}_{\mu}^{\nu}$ or with $\mathcal{W}_{\mu}^{\nu}$; note that $\mathcal{J}_{i}$ (which turns out to be subleading in the long-wavelength approximation) is the same both in the Landau-Lifshitz and in the Ford-Parker parametrizations.

${ }^{6}$ Note that, in spite of the parametrization, $\left\langle\Pi_{i j}\right\rangle=0$ since the anisotropic stress is fully inhomogeneous.
} 
Conversely the inhomogeneities induced by the longwavelength gravitons may affect the curvature perturbations after inflation: during the radiation-dominated phase the long-wavelength modes decrease more slowly than the background energy density (going as $a^{-4}$ in the conventional post-inflationary thermal history). With these specifications, and recalling that $h_{i j}$ is gauge invariant for infinitesimal coordinate transformations preserving the tensor nature of the fluctuation, we can consider the scalar modes of the geometry in the presence of the fluctuations of the energy density and pressure induced by the relic gravitons. Using Eq. (3.1), the (00) and (0i) components of Eq. (2.5) give the Hamiltonian and the momentum constraints whose explicit form is given by

$$
\begin{gathered}
\nabla^{2} \Psi-3 \mathcal{H}\left(\Psi^{\prime}+\mathcal{H} \Phi\right)=\frac{a^{2} \ell_{\mathrm{P}}^{2}}{2}\left[\delta \rho_{\mathrm{t}}+\delta \rho_{\mathrm{gw}}\right], \\
\nabla^{2}\left(\Psi^{\prime}+\mathcal{H} \Phi\right)=-\frac{a^{2} \ell_{\mathrm{P}}^{2}}{2}\left[\left(p_{\mathrm{t}}+\rho_{\mathrm{t}}\right) \theta_{\mathrm{t}}+\vec{\nabla} \cdot \overrightarrow{\mathcal{J}}\right],
\end{gathered}
$$

where $\theta_{\mathrm{t}}$ is, as usual, the three-divergence of the total velocity field while $\Phi$ and $\Psi$ are the gauge-invariant Bardeen potentials [14]; $\delta \rho_{\mathrm{t}}$ and $\delta p_{\mathrm{t}}$ denote the scalar fluctuations of the total energy density and total pressure of the fluid during the post-inflationary phase. The $(i=j)$ and $(i \neq j)$ components of Eq. (2.5) read instead

$$
\begin{gathered}
\Psi^{\prime \prime}+\mathcal{H}\left(\Phi^{\prime}+2 \Psi^{\prime}\right)+\left(\mathcal{H}^{2}+2 \mathcal{H}^{\prime}\right) \Phi+\frac{1}{3} \nabla^{2}(\Phi-\Psi) \\
=\frac{a^{2} \ell_{\mathrm{P}}^{2}}{2}\left(\delta p_{\mathrm{t}}+\delta p_{\mathrm{gw}}\right), \\
\nabla^{2}(\Phi-\Psi)=\frac{3}{2} \ell_{\mathrm{P}}^{2} a^{2} \Pi_{\mathrm{gw}},
\end{gathered}
$$

where the practical notation

$$
\partial_{i} \partial_{j} \Pi^{i j}=\nabla^{2} \Pi_{\mathrm{gw}}
$$

has been introduced. In connection with the anisotropic stress we remark that all sources of anisotropic stress besides the relic gravitons have been neglected but this will not alter the conclusions of the analysis.

\section{B. Curvature perturbations}

The evolution of the curvature perturbations can be easily obtained by introducing the total sound speed of the system, i.e. $c_{\mathrm{st}}^{2}=p_{\mathrm{t}}^{\prime} / \rho_{\mathrm{t}}^{\prime}$. By multiplying Eq. (3.3) by $c_{\mathrm{st}}^{2}$ and subtracting the obtained result from Eq. (3.5) we get to the relation

$$
\begin{gathered}
\Psi^{\prime \prime}+\mathcal{H}\left[\Phi^{\prime}+\left(2+3 c_{\mathrm{st}}^{2}\right) \Psi^{\prime}\right]+\left[\mathcal{H}^{2}\left(1+3 c_{\mathrm{st}}^{2}\right)+2 \mathcal{H}^{\prime}\right] \Phi \\
-c_{\mathrm{st}}^{2} \nabla^{2} \Psi+\frac{1}{3} \nabla^{2}(\Phi-\Psi) \\
=\frac{a^{2} \ell_{\mathrm{P}}^{2}}{2}\left[\delta p_{\mathrm{nad}}+\delta p_{\mathrm{gw}}-c_{\mathrm{st}}^{2} \delta \rho_{\mathrm{gw}}\right] .
\end{gathered}
$$

The standard notation $\delta p_{\text {nad }}=\delta p_{\mathrm{t}}-c_{\mathrm{st}}^{2} \delta \rho_{\mathrm{t}}$ has been introduced in Eq. (3.8). In the conventional adiabatic scenario (where nonadiabatic modes are, by definition, absent) we have $\delta p_{\text {nad }}=0$.

From the gauge-invariant expression of the curvature perturbations $^{7}$ on comoving orthogonal hypersurfaces [14] (see also Ref. [15]) we obtain the relation

$$
\begin{aligned}
& \mathcal{R}^{\prime}=\Sigma_{\mathcal{R}}-\frac{2 \mathcal{H} c_{\mathrm{st}}^{2} \nabla^{2} \Psi}{\ell_{\mathrm{P}}^{2} a^{2}\left(\rho_{\mathrm{t}}+p_{\mathrm{t}}\right)} \\
& \Sigma_{\mathcal{R}}=-\frac{\mathcal{H}}{p_{\mathrm{t}}+\rho_{\mathrm{t}}} \delta p_{\mathrm{nad}}+\frac{\mathcal{H}}{p_{\mathrm{t}}+\rho_{\mathrm{t}}}\left[c_{\mathrm{st}}^{2} \delta \rho_{\mathrm{gw}}-\delta p_{\mathrm{gw}}+\Pi_{\mathrm{gw}}\right] .
\end{aligned}
$$

Equation (3.9) is not decoupled since it depends on $\Psi$. By taking the first derivative of both sides of Eq. (3.9) and by using Eq. (3.6) in the obtained expression we obtain the following evolution equation:

$\mathcal{R}^{\prime \prime}+2 \frac{z_{\mathrm{t}}^{\prime}}{z_{\mathrm{t}}} \mathcal{R}^{\prime}-c_{\mathrm{st}}^{2} \nabla^{2} \mathcal{R}=\Sigma_{\mathcal{R}}^{\prime}+2 \frac{z_{\mathrm{t}}^{\prime}}{z_{\mathrm{t}}} \Sigma_{\mathcal{R}}+\frac{3 a^{4}}{z_{\mathrm{t}}^{2}} \Pi_{\mathrm{gw}}$,

where $z_{\mathrm{t}}$ is defined as $z_{\mathrm{t}}=a^{2} \sqrt{p_{\mathrm{t}}+\rho_{\mathrm{t}}} /\left(c_{\mathrm{st}} \mathcal{H}\right)$. To derive Eq. (3.11) we need to use the evolution equations of the background written in the conformally flat case, i.e.

$3 \mathcal{H}^{2}=\ell_{\mathrm{P}}^{2} a^{2} \rho_{t}, \quad 2\left(\mathcal{H}^{2}-\mathcal{H}^{\prime}\right)=\ell_{\mathrm{P}}^{2} a^{2}\left(\rho_{t}+p_{t}\right) ;$

by combining Eqs. (3.12) we get, as expected, the result coming from the covariant conservation of the energymomentum tensor of the sources, i.e. $\rho_{t}^{\prime}+3 \mathcal{H}\left(\rho_{t}+p_{t}\right)=0$. We must recall that the possible effect of the energy density of the gravitons on the evolution of the background has been totally neglected due to its smallness. Relic gravitons may instead contribute to the evolution of the curvature perturbations as Eq. (3.11) shows.

It is useful to conclude this discussion by stressing the connection of $\mathcal{R}$ with $\zeta$ which denotes the total density contrast on uniform-curvature hypersurfaces. The full gauge-invariant definition of $\zeta$ can be given in terms of the gauge-invariant Bardeen potential and it is given by $\zeta=$ $-\Psi-\mathcal{H}\left(\delta \rho_{\mathrm{t}}+\delta \rho_{\mathrm{gw}}\right) / \rho_{\mathrm{t}}^{\prime}$. Recalling then the definition of $\mathcal{R}$ given above [see above Eq. (3.9)], Eq. (3.3) demands that

$$
\zeta=\mathcal{R}+\frac{2 \nabla^{2} \Psi}{3 \ell_{\mathrm{P}}^{2}\left(\rho_{\mathrm{t}}+p_{\mathrm{t}}\right)} .
$$

\footnotetext{
${ }^{7}$ We recall that $\mathcal{R}$ is defined as $\mathcal{R}=-\Psi-\mathcal{H}\left(\mathcal{H} \Phi+\Psi^{\prime}\right) /$ $\left(\mathcal{H}^{2}-\mathcal{H}^{\prime}\right)$
} 
Equation (3.13) shows that the decoupled equation obeyed by $\zeta$ coincides with Eq. (3.11) only in the large-scale limit.

\section{Large-scale solutions with relic gravitons}

Equation (3.11) will now be studied for typical wavelengths larger than the Hubble radius. In this case the term $c_{\mathrm{st}}^{2} \nabla^{2} \mathcal{R}$ can be consistently dropped but it is appropriate to stress that it would be incorrect to eliminate the contribution of the gradients directly in Eq. (3.9). By dropping $\nabla^{2} \Psi$ we would also neglect terms that are comparable to the contribution of the anisotropic stress of the relic gravitons. We could neglect the presence of any nonadiabatic mode in the fluid sector by setting $\delta p_{\text {nad }}=0$ in Eq. (3.10). However this assumption is not necessary insofar as we will write down a general solution containing also the first derivative of the curvature perturbations.

With the previous specifications, Eq. (3.11) can be first written in the form of an integral equation as

$$
\begin{aligned}
\mathcal{R}(\vec{x}, \tau)= & \mathcal{R}_{*}(\vec{x})+z_{\mathrm{t}}^{2}\left(\tau_{1}\right)\left(\mathcal{R}^{\prime}-\Sigma_{\mathcal{R}}\right)_{\tau_{1}} \\
& \times \int_{\tau_{1}}^{\tau} \frac{d \tau^{\prime}}{z_{\mathrm{t}}^{2}\left(\tau^{\prime}\right)}+\int_{\tau_{1}}^{\tau} \Sigma_{\mathcal{R}}\left(\vec{x}, \tau^{\prime}\right) d \tau^{\prime} \\
& +3 \int_{\tau_{1}}^{\tau} \frac{d \tau^{\prime}}{z_{\mathrm{t}}^{2}\left(\tau^{\prime}\right)} \int_{\tau_{1}}^{\tau^{\prime}} a^{4}\left(\tau^{\prime \prime}\right) \Pi_{\mathrm{gw}}\left(\vec{x}, \tau^{\prime \prime}\right) d \tau^{\prime \prime} \\
& +\int_{\tau_{1}}^{\tau} \frac{d \tau^{\prime}}{z_{\mathrm{t}}^{2}\left(\tau^{\prime}\right)} \int_{\tau_{1}}^{\tau^{\prime}} z_{\mathrm{t}}^{2}\left(\tau^{\prime \prime}\right) c_{\mathrm{st}}^{2}\left(\tau^{\prime \prime}\right) \nabla^{2} \mathcal{R}\left(\vec{x}, \tau^{\prime \prime}\right) d \tau^{\prime \prime},
\end{aligned}
$$

which can be formally solved by iteration in the longwavelength limit where the term $c_{\mathrm{st}}^{2} \nabla^{2} \mathcal{R}_{*}$ can be neglected in comparison with the other contributions. Thus the longwavelength solution of Eq. (3.11) can be written,

$$
\begin{aligned}
\mathcal{R}(\vec{x}, \tau)= & \mathcal{R}_{*}(\vec{x})+z_{\mathrm{t}}^{2}\left(\tau_{1}\right)\left(\mathcal{R}^{\prime}-\Sigma_{\mathcal{R}}\right)_{\tau_{1}} \\
& \times \int_{\tau_{1}}^{\tau} \frac{d \tau^{\prime}}{z_{\mathrm{t}}^{2}\left(\tau^{\prime}\right)}+\int_{\tau_{1}}^{\tau} \Sigma_{\mathcal{R}}\left(\vec{x}, \tau^{\prime}\right) d \tau^{\prime} \\
& +3 \int_{\tau_{1}}^{\tau} \frac{d \tau^{\prime}}{z_{\mathrm{t}}^{2}\left(\tau^{\prime}\right)} \int_{\tau_{1}}^{\tau^{\prime}} a^{4}\left(\tau^{\prime \prime}\right) \Pi_{\mathrm{gw}}\left(\vec{x}, \tau^{\prime \prime}\right) d \tau^{\prime \prime} \\
& +\mathcal{O}\left(c_{\mathrm{st}}^{2} \nabla^{2} \mathcal{R}_{*}\right) .
\end{aligned}
$$

The solution reported in Eq. (3.14) has been written in general without assuming anything about the adiabatic (or nonadiabatic) nature of the dominant source of inhomogeneity. In what follows we shall focus however on the case where $\delta p_{\text {and }}=0$. Note that Eq. (3.14) is written in real space but it can be easily translated to Fourier space. Before proceeding further we need to compute the evolution of the various terms appearing inside the integrals. These will be the main themes of the forthcoming Sec. IV.

\section{POWER SPECTRA AND EXPLICIT ESTIMATES}

The calculations of the various power spectra present similar technical aspects so we shall discuss the case of the energy density and then mention the salient results for the pressure and the anisotropic stress. To avoid digressions a number of explicit results have been relegated to Appendices A, B and C.

\section{A. Power spectrum of the energy density of the relic gravitons}

Let us therefore go back to the fluctuations of the energy density defined in Eq. (3.2) and introduce their Fourier transform

$$
\delta \rho_{\mathrm{gw}}(\vec{x}, \tau)=\frac{1}{(2 \pi)^{3 / 2}} \int d^{3} k e^{-i \vec{q} \cdot \vec{x}} \delta \rho_{\mathrm{gw}}(\vec{q}, \tau),
$$

whose corresponding power spectrum is defined, within the present conventions, as

$$
\left\langle\delta \rho_{\mathrm{gw}}(\vec{p}, \tau) \delta \rho_{\mathrm{gw}}(\vec{q}, \tau)\right\rangle=\frac{2 \pi^{2}}{q^{3}} \delta^{(3)}(\vec{q}+\vec{p}) \mathcal{P}_{\rho_{\mathrm{gw}}}(q, \tau)
$$

According to the definition (3.2), $\left\langle\delta \rho_{\mathrm{gw}}(\vec{q}, \tau)\right\rangle=0$; moreover Eq. (2.17) (in the Ford-Parker parametrization) and Eq. (2.11) (in the Landau-Lifshitz parametrization) lead, in Fourier space, to the following results:

$$
\begin{aligned}
\delta \rho_{\mathrm{gw}}^{(F P)}(\vec{q}, \tau)= & \frac{1}{8 \ell_{\mathrm{P}}^{2} a^{2}} \int \frac{d^{3} k}{(2 \pi)^{3 / 2}}\left\{\hat{H}_{\ell m}(\vec{k}, \tau) \hat{H}_{\ell m}(\vec{q}-\vec{k}, \tau)\right. \\
& +[\vec{k} \cdot(\vec{k}-\vec{q})] \hat{h}_{\ell m}(\vec{k}, \tau) \hat{h}_{\ell m}(\vec{q}-\vec{k}, \tau) \\
& \left.-\frac{2 \pi^{2}}{k^{3}}\left[\mathcal{P}_{g g}(k, \tau)+k^{2} \mathcal{P}_{f f}(k, \tau)\right] \delta^{(3)}(\vec{q})\right\},
\end{aligned}
$$

$$
\begin{aligned}
\delta \rho_{\mathrm{gw}}^{(L L)}(\vec{q}, \tau)= & \frac{1}{8 \ell_{\mathrm{P}}^{2} a^{2}} \int \frac{d^{3} k}{(2 \pi)^{3 / 2}}\left\{\hat{H}_{\ell m}(\vec{k}, \tau) \hat{H}_{\ell m}(\vec{q}-\vec{k}, \tau)+[\vec{k} \cdot(\vec{k}-\vec{q})] \hat{h}_{\ell m}(\vec{k}, \tau) \hat{h}_{\ell m}(\vec{q}-\vec{k}, \tau)\right. \\
& +4 \mathcal{H}\left[\hat{H}_{\ell m}(\vec{k}, \tau) \hat{h}_{\ell m}(\vec{q}-\vec{k}, \tau)+\hat{h}_{\ell m}(\vec{k}, \tau) \hat{H}_{\ell m}(\vec{q}-\vec{k}, \tau)\right]-\frac{2 \pi^{2}}{k^{3}}\left[\mathcal{P}_{g g}(k, \tau)+k^{2} \mathcal{P}_{f f}(k, \tau)\right. \\
& \left.\left.+4 \mathcal{H}\left(\mathcal{P}_{f g}(k, \tau)+\mathcal{P}_{g f}(k, \tau)\right)\right] \delta^{(3)}(\vec{q})\right\},
\end{aligned}
$$

where, according to Eq. (2.15), the notation $\hat{H}_{\ell m}(\vec{k}, \tau)=\hat{h}_{\ell m}^{\prime}(\vec{k}, \tau)$ has been introduced. 
The power spectra of the fluctuation defined in Eq. (4.2) can be written, with the help of Eqs. (4.3) and (4.4), as

$$
\begin{aligned}
\mathcal{P}_{\rho_{\mathrm{gw}}}^{(F P)}(q, \tau)= & \frac{q^{3}}{128 \pi \ell_{\mathrm{P}}^{4} a^{4}(\tau)} \int \frac{d^{3} k}{k^{3}|\vec{q}-\vec{k}|^{3}} \\
& \times \mathcal{Q}(\vec{k}, \vec{q})\left\{\mathcal{P}_{g g}(k, \tau) \mathcal{P}_{g g}(|\vec{q}-\vec{k}|, \tau)\right. \\
& +\vec{k} \cdot(\vec{q}-\vec{k})\left[\mathcal{P}_{f g}(k, \tau) \mathcal{P}_{g f}(|\vec{q}-\vec{k}|, \tau)\right. \\
& \left.+\mathcal{P}_{g f}(k, \tau) \mathcal{P}_{f g}(|\vec{q}-\vec{k}|, \tau)\right] \\
& \left.+[\vec{k} \cdot(\vec{q}-\vec{k})]^{2} \mathcal{P}_{f f}(k, \tau) \mathcal{P}_{f f}(|\vec{q}-\vec{k}|, \tau)\right\},
\end{aligned}
$$

$$
\begin{aligned}
\mathcal{P}_{\rho_{\mathrm{gw}}}^{(L L)}(q, \tau)= & \frac{q^{3}}{128 \pi \ell_{\mathrm{P}}^{4} a^{4}(\tau)} \\
& \times \int \frac{d^{3} k}{k^{3}|\vec{q}-\vec{k}|^{3}} \mathcal{Q}(\vec{k}, \vec{q})\left[16 \mathcal{H}^{2} \mathcal{A}_{1}(\vec{k}, \vec{q})\right. \\
& \left.+4 \mathcal{H} \mathcal{A}_{2}(\vec{k}, \vec{q})+\mathcal{A}_{3}(\vec{k}, \vec{q})\right]
\end{aligned}
$$

the functions $\mathcal{A}_{1}(\vec{k}, \vec{q}, \tau), \mathcal{A}_{2}(\vec{k}, \vec{q}, \tau)$ and $\mathcal{A}_{3}(\vec{k}, \vec{q}, \tau)$ are defined in Eqs. (A1), (A2) and (A3) of Appendix A. Note that $\mathcal{Q}(\vec{k}, \vec{q})$ can be computed thanks to the identity given in Eq. (2.31) by identifying $\hat{a}=\hat{k}$ and $\hat{b}=(\vec{q}-\vec{k}) /|\vec{q}-\vec{k}|$ :

$\mathcal{Q}(\vec{k}, \vec{q})=\frac{1}{16}\left[1+4 \frac{\left(\vec{k} \cdot \vec{q}-k^{2}\right)^{2}}{k^{2}|\vec{q}-\vec{k}|^{2}}+3 \frac{\left(\vec{k} \cdot \vec{q}-k^{2}\right)^{4}}{k^{4}|\vec{q}-\vec{k}|^{4}}\right]$.

The presence of additional terms in Eq. (4.4) complicates the correlator and it is ultimately responsible for the form of the power spectrum of Eq. (4.6). Thus the differences between Eqs. (4.5) and (4.6) are a direct consequence of the form of Eqs. (4.3) and (4.4). We remark that Eqs. (4.5) and (4.6) have been written in general but $P_{f f}(k, \tau)$, $P_{g g}(k, \tau), P_{f g}(k, \tau)$ and $P_{g f}(k, \tau)$ depend on the mode functions according to Eqs. (2.28) and (2.29). The mode functions are determined, in their turn, by the evolution of the background that will now be specified.

\section{B. Explicit forms for the mode functions}

We shall be dealing hereafter with the following analytical form of the scale factor ${ }^{8}$ :

$$
a(\tau)=-\frac{\tau_{1}}{\tau}, \quad \tau \leq-\tau_{1},
$$

\footnotetext{
${ }^{8}$ Equations (4.8) and (4.9) are continuous (with their first derivatives) in $\tau=-\tau_{1}$. Similarly Eqs. (4.9) and (4.10) are continuous (with their first derivatives) in $\tau=\tau_{2}$.
}

$$
\begin{aligned}
& a(\tau)=\frac{\tau+2 \tau_{1}}{\tau_{1}}, \quad-\tau_{1}<\tau<\tau_{2}, \\
& a(\tau)=\frac{\left(\tau+\tau_{2}+4 \tau_{1}\right)^{2}}{4 \tau_{1}\left(\tau_{2}+2 \tau_{1}\right)}, \quad \tau>\tau_{2} .
\end{aligned}
$$

The typical time scale $\tau_{1}$ denotes the transition from the de Sitter stage of expansion to the radiation-dominated epoch ending at the conformal time $\tau_{2}$ which coincides with the equality time and defines the beginning of the matterdominated period of expansion.

Equation (4.8) implies that the mode function solving Eq. (2.10) for $\tau \leq-\tau_{1}$ is

$$
\begin{aligned}
f_{k}(\tau) & =\frac{1}{\sqrt{2 k}}\left(1-\frac{i}{x}\right) e^{-i x}, \\
f_{k}^{\prime}(\tau) & =i \sqrt{\frac{k}{2}}\left[\frac{1}{x^{2}}-1+\frac{i}{x}\right] e^{-i x},
\end{aligned}
$$

where we introduced the dimensionless combination $x=k \tau$. For $\tau>-\tau_{1}$ the continuity of the canonical field operators demands the continuity of the mode functions, i.e.

$$
\begin{aligned}
& f_{k}\left(-\tau_{1}\right)=c_{+}(k) F_{k}\left(-\tau_{1}\right)+c_{-}(k) F_{k}^{*}\left(-\tau_{1}\right), \\
& f_{k}^{\prime}\left(-\tau_{1}\right)=c_{+}(k) F_{k}^{\prime}\left(-\tau_{1}\right)+c_{-}(k) F_{k}^{\prime *}\left(-\tau_{1}\right),
\end{aligned}
$$

where $F_{k}(\tau)$ and $F_{k}^{\prime}(\tau)$ are now given by

$$
\begin{aligned}
& F_{k}(\tau)=\frac{1}{\sqrt{2 k}} e^{-i\left(x+2 x_{1}\right)}, \\
& F_{k}^{\prime}(\tau)=-i \sqrt{\frac{k}{2}} e^{-i\left(x+2 x_{1}\right)},
\end{aligned}
$$

and solve Eq. (2.10) during the radiation-dominated stage [see Eq. (4.9)]. Inserting Eqs. (4.11) and (4.13) into Eq. (4.12), $c_{ \pm}\left(x_{1}\right)$ are determined to be ${ }^{9}$

$c_{+}\left(x_{1}\right)=\frac{e^{2 i x_{1}}\left(2 x_{1}^{2}-1+2 i x_{1}\right)}{2 x_{1}^{2}}, \quad c_{-}\left(x_{1}\right)=\frac{1}{2 x_{1}^{2}}$,

where, following the same notation as in Eq. (4.11), $x_{1}=k \tau_{1}$. Recalling Eqs. (2.28) and (2.29) and using Eq. (4.12) all the power spectra appearing in Eq. (4.5) can be written in more explicit terms.

The general formulas are rather lengthy and will not be reported; however by focusing on a single illustrative combination we shall have expressions of the following kind:

\footnotetext{
${ }^{9}$ As expected, it follows from Eq. (4.14) that $\left|c_{+}\left(x_{1}\right)\right|^{2}-$ $\left|c_{-}\left(x_{1}\right)\right|^{2}=1$.
} 


$$
\begin{aligned}
& \mathcal{P}_{g g}(k, \tau)+k^{2} \mathcal{P}_{f f}(k, \tau) \\
& =\frac{4 \ell_{\mathrm{P}}^{2} k^{3}}{\pi^{2} a^{2}(\tau)}\left\{\left(2\left|c_{-}\left(x_{1}\right)\right|^{2}+1\right)\left[\left|G_{k}\left(x, x_{1}\right)\right|^{2}+k^{2}\left|F_{k}\left(x, x_{1}\right)\right|^{2}\right]\right. \\
& \quad+c_{-}\left(x_{1}\right) c_{+}^{*}\left(x_{1}\right)\left[G_{k}^{* 2}\left(x, x_{1}\right)+k^{2} F_{k}^{* 2}\left(x, x_{1}\right)\right] \\
& \left.\quad+c_{-}^{*}\left(x_{1}\right) c_{+}\left(x_{1}\right)\left[G_{k}^{2}\left(x, x_{1}\right)+k^{2} F_{k}^{2}\left(x, x_{1}\right)\right]\right\}
\end{aligned}
$$

and so on for the other quadratic and quartic combinations appearing in Eqs. (4.5) and (4.6). Note that, in Eq. (4.15) $G_{k}(\tau)=F_{k}^{\prime}(\tau)-\mathcal{H} F_{k}(\tau)$, in analogy with the notation employed for $g_{k}(\tau)$ in Eq. (2.26).

Equation (4.14) applies when the relevant modes exit the Hubble radius during the inflationary phase and reenter in the radiation epoch. Conversely, if the corresponding wavelengths are still larger than the Hubble radius after matter-radiation equality, then the mode functions of Eq. (4.13) should be substituted by

$$
\begin{aligned}
F_{k}(\tau)= & \frac{1}{\sqrt{2 k}}\left(1+\frac{i}{x+x_{2}+2 x_{1}}\right) e^{i\left(x+x_{2}+2 x_{1}\right)}, \\
G_{k}(\tau)= & \sqrt{\frac{k}{2}}\left(i-\frac{3}{\left(x+x_{2}+2 x_{1}\right)}\right. \\
& \left.-\frac{3 i}{\left(x+x_{2}+2 x_{1}\right)^{2}}\right) e^{i\left(x+x_{2}+2 x_{1}\right)}
\end{aligned}
$$

where $x_{2}=k \tau_{2}$ and $\tau_{2}$ coincides with the equality time; Eq. (4.16) solves Eq. (2.10) for $\tau>\tau_{2}$ [see Eq. (4.10)]. If $x_{1} \ll x_{2} \leq 1$, the corresponding wavelength is about to re-enter during the matter-dominated epoch. If $x_{1} \ll 1$ and $x_{2} \ll 1$ the relevant wavelengths are larger than the Hubble radius both during the radiation-dominated epoch and during the matter-dominated phase. The coefficients $d_{ \pm}\left(x_{1}, x_{2}\right)$ [obeying, $\left|d_{+}\left(x_{1}, x_{2}\right)\right|^{2}-\left|d_{-}\left(x_{1}, x_{2}\right)\right|^{2}=1$ ] valid for $\tau>\tau_{2}$ are

$$
\begin{aligned}
d_{+}\left(x_{1}, x_{2}\right)= & \frac{1}{8\left(x_{2}+2 x_{1}\right)^{2}}\left[-1-4 i\left(x_{2}+2 x_{1}\right)\right. \\
& \left.+8\left(x_{2}+2 x_{1}\right)^{2}\right] e^{-i\left(x_{2}+2 x_{1}\right)}, \\
d_{-}\left(x_{1}, x_{2}\right)= & -\frac{1}{8\left(x_{2}+2 x_{1}\right)^{2}} e^{3 i\left(x_{2}+2 x_{1}\right)} .
\end{aligned}
$$

We shall now illustrate the expansion required for the explicit evaluation of the power spectra of the energy density, of the pressure and of the anisotropic stress. Thanks to Eqs. (4.12) and (4.14) the following double expansion holds when the wavelengths are larger than the Hubble radius at the end of inflation (i.e. $x_{1} \ll 1$ ) and later on (i.e. $x \ll 1$ ):

$$
\begin{gathered}
\frac{\left|g_{k}\left(x, x_{1}\right)\right|^{2}}{k}=\frac{1}{18 x_{1}^{4}}\left[x^{4}+\mathcal{O}\left(x^{6}\right)\right] \\
+\frac{2}{3 x_{1}^{3}}\left[x+\mathcal{O}\left(x^{3}\right)\right]+\mathcal{O}\left(x_{1}^{-2}\right) \\
f_{k}\left(x, x_{1}\right) g_{k}^{*}\left(x, x_{1}\right)+f_{k}^{*}\left(x, x_{1}\right) g_{k}\left(x, x_{1}\right) \\
=-\frac{1}{3 x_{1}^{4}}\left[x^{3}+\mathcal{O}\left(x^{5}\right)\right] \\
-\frac{2}{x_{1}^{3}}\left[1+\frac{2}{3} x^{2}+\mathcal{O}\left(x^{4}\right)\right]+\mathcal{O}\left(x_{1}^{-2}\right) \\
k\left|f_{k}\left(x, x_{1}\right)\right|^{2}=\frac{1}{2 x_{1}^{4}}\left[x^{2}-\frac{x^{4}}{3}+\mathcal{O}\left(x^{5}\right)\right] \\
+\frac{2}{x_{1}^{3}}\left[x-\frac{2}{3} x^{3}+\mathcal{O}\left(x^{5}\right)\right]+\mathcal{O}\left(x_{1}^{-2}\right),
\end{gathered}
$$

where the symbol $\mathcal{O}(\ldots)$ defines the order of magnitude of the subleading terms. On the basis of Eqs. (4.18), (4.19) and (4.20) the hierarchy between the different contributions can be gauged since, by definition, not only is $x_{1} \ll 1$ and $x \ll 1$, but also $x_{1} \ll x$. Thus, from Eqs. (4.18)-(4.20), we shall have that

$$
\begin{aligned}
\frac{\left|g_{k}\left(x, x_{1}\right)\right|^{2}}{k} & \ll\left[f_{k}\left(x, x_{1}\right) g_{k}^{*}\left(x, x_{1}\right)+f_{k}^{*}\left(x, x_{1}\right) g_{k}\left(x, x_{1}\right)\right] \\
& \ll k\left|f_{k}\left(x, x_{1}\right)\right|^{2} .
\end{aligned}
$$

The results of Eqs. (4.18)-(4.20) and (4.21) can be used to expand more complicated combinations of mode functions appearing in Eqs. (4.5) and (4.6) in powers of $k \tau_{1} \ll 1$ and of $|\vec{q}-\vec{k}| \tau_{1} \ll 1$. For illustration we report the leading terms of the expansion for some relevant contributions:

$$
\begin{aligned}
\left|g_{k}(\tau)\right|^{2}\left|g_{|\vec{q}-\vec{k}|}(\tau)\right|^{2} & \simeq \frac{k|\vec{q}-\vec{k}|}{324}\left(\frac{\tau}{\tau_{1}}\right)^{8}, \\
\left|f_{k}(\tau)\right|^{2}\left|f_{|\vec{q}-\vec{k}|}(\tau)\right|^{2} & \simeq \frac{1}{4 k^{3}|\vec{q}-\vec{k}|^{3}} \frac{\tau^{2}}{\tau_{1}^{4}}, \\
f_{k}(\tau) g_{k}^{*}(\tau) f_{|\vec{q}-\vec{k}|}(\tau) g_{|\vec{q}-\vec{k}|}^{*}(\tau) & \simeq \frac{1}{36 k|\vec{q}-\vec{k}|} \frac{\tau^{3}}{\tau_{1}^{4}} .
\end{aligned}
$$

The results of Eq. (4.22) have been derived by introducing the four dimensionless variables

$$
\begin{aligned}
x_{1} & =k \tau_{1} \ll 1, & \tilde{x}_{1} & =|\vec{q}-\vec{k}| \tau_{1} \ll 1, \\
x & =k \tau \ll 1, & \tilde{x} & =|\vec{q}-\vec{k}| \tau \ll 1,
\end{aligned}
$$

and by enforcing the conditions $x_{1} \ll x \ll 1$ and $\tilde{x}_{1} \ll$ $\tilde{x} \ll 1$. The results of Eq. (4.22) then follow rather easily 
by expressing the leading results of the expansion in terms of the original variables, i.e. $(k,|\vec{q}-\vec{k}|)$ and $\left(\tau, \tau_{1}\right)$.

\section{Asymptotic expressions of the power spectra}

Equations (4.5) and (4.6) can now be evaluated in the interesting physical limits (for instance $x_{1} \ll x \ll 1$ and $\left.\tilde{x}_{1} \ll \tilde{x} \ll 1\right)$ by using the results of Eqs. (4.21) and (4.22)(4.23). The leading-order results for the expansion are

$$
\mathcal{P}_{\rho_{\mathrm{gw}}}^{(F P)}(q, \tau) \simeq \frac{H^{8}}{512 \pi^{5} a^{4}(\tau)}\left|q \tau_{1}\right|^{4} \mathcal{F}_{\rho_{\mathrm{gw}}}^{(F P)}(q),
$$

$$
\mathcal{P}_{\rho_{\mathrm{gw}}}^{(L L)}(q, \tau) \simeq \frac{H^{8}}{512 \pi^{5} a^{4}(\tau)}\left|q \tau_{1}\right|^{4} \mathcal{F}_{\rho_{\mathrm{gw}}}^{(L L)}(q),
$$

where $\mathcal{F}_{\rho_{\mathrm{gw}}}^{(F P)}(q)$ and $\mathcal{F}_{\rho_{\mathrm{gw}}}^{(L L)}(q)$ are defined as

$$
\begin{aligned}
\mathcal{F}_{\rho_{\mathrm{gw}}}^{(F P)}(q)= & \int d^{3} k \frac{\left[\vec{k} \cdot \vec{q}-k^{2}\right]^{2}\left[k^{2}|\vec{q}-\vec{k}|^{2}+3\left(\vec{k} \cdot q-k^{2}\right)^{2}\right]\left[k^{2}|\vec{q}-\vec{k}|^{2}+\left(\vec{k} \cdot q-k^{2}\right)^{2}\right]}{k^{7} q|\vec{q}-\vec{k}|^{7}}, \\
\mathcal{F}_{\rho_{\mathrm{gw}}}^{(L L)}(q)= & \int d^{3} k \frac{\left[k^{2}|\vec{q}-\vec{k}|^{2}+3\left(\vec{k} \cdot \vec{q}-k^{2}\right)^{2}\right]\left[k^{2}|\vec{q}-\vec{k}|^{2}+\left(\vec{k} \cdot q-k^{2}\right)^{2}\right]}{q k^{7}|\vec{q}-\vec{k}|^{7}}\left[\left|\vec{k} \cdot \vec{q}-k^{2}\right|^{2}\right. \\
& \left.+\frac{16}{9}\left(|\vec{q}-\vec{k}|^{4}+\frac{16}{9} k^{4}\right)+\frac{32}{9} k^{2}|\vec{q}-\vec{k}|^{2}-\frac{4}{3} \vec{k} \cdot(\vec{q}-\vec{k})\left(k^{2}+|\vec{q}-\vec{k}|^{2}\right)\right] .
\end{aligned}
$$

Both $\mathcal{F}_{\rho_{\mathrm{gw}}}^{(F P)}(q)$ and $\mathcal{F}_{\rho_{\mathrm{gw}}}^{(L L)}(q)$ are dimensionless. Let us now pause for a moment and remark that Eqs. (4.24) and (4.25) are a direct consequence of Eqs. (4.3) and (4.4). In spite of the different forms of $\mathcal{F}_{\rho_{\mathrm{gw}}}^{(L L)}(q)$ and $\mathcal{F}_{\rho_{\mathrm{gw}}}^{(F P)}(q)$ their contributions to the spectrum of the energy density are of comparable magnitude. The integrals appearing in Eq. (4.26) can be written, in a more explicit form, as

$$
\begin{aligned}
& \mathcal{F}_{\rho_{\mathrm{gw}}}^{(F P)}(q)=2 \pi \int_{-1}^{1} d y \int_{k_{\text {min }}}^{k_{\max }} \frac{k d k}{q} \mathcal{L}(k, q, y)(q y-k)^{2}, \\
& \mathcal{F}_{\rho_{\mathrm{gw}}}^{(L L)}(q)=\frac{2 \pi}{9} \int_{-1}^{1} d y \int_{k_{\text {min }}}^{k_{\max }} \frac{d k}{q k} \mathcal{L}(k, q, y) \mathcal{M}(k, q, y),
\end{aligned}
$$

where the functions $\mathcal{L}(k, q, y)$ and $\mathcal{M}(k, q, y)$ are defined as

$$
\begin{aligned}
\mathcal{L}(k, q, y) & =\frac{\left[4 k^{2}-8 q k y+q^{2}\left(1+3 y^{2}\right)\right]\left[2 k^{2}-4 q k y+q^{2}\left(1+y^{2}\right)\right]}{\left(q^{2}+k^{2}-2 q k y\right)^{7 / 2}}, \\
\mathcal{M}(k, q, y) & =\left[49 k^{4}+16 q^{4}+k^{2} q^{2}\left(49 y^{2}+52\right)-52 q^{3} k y-62 q k^{3} y\right] .
\end{aligned}
$$

After performing the explicit integration over $y$ in Eqs. (4.27) and (4.28), the following results can be obtained:

$$
\begin{aligned}
& \mathcal{F}_{\rho_{\mathrm{gw}}}^{(F P)}(q)=\frac{8 \pi}{105} \int_{k_{\min }}^{k_{\max }} \frac{d k}{k^{6} q^{2}}\left[\mathcal{N}_{+}^{(F P)}(k, q)|k+q|+\mathcal{N}_{-}^{(F P)}(k, q)|k-q|\right], \\
& \mathcal{F}_{\rho_{\mathrm{gw}}}^{(L L)}(q)=\frac{8 \pi}{135} \int_{k_{\min }}^{k_{\max }} \frac{d k}{k^{6} q^{2}} \frac{\mathcal{N}_{-}^{(L L)}(k, q)|k-q|+\mathcal{N}_{+}^{(L L)}(k, q)|k+q|}{|k+q||k-q|},
\end{aligned}
$$

where $\mathcal{N}_{ \pm}^{(F P)}(k, q)$ and $\mathcal{N}_{ \pm}^{(L L)}(k, q)$ are given, respectively, by ${ }^{10}$

$$
\begin{aligned}
\mathcal{N}_{ \pm}^{(F P)}(q)= & \mp 41 k^{6}+169 k^{5} q \mp 169 k^{4} q^{2}-146 k^{3} q^{3} \mp 146 k^{2} q^{4}+36 k q^{5} \mp 36 q^{6} \\
\mathcal{N}_{ \pm}^{(L L)}(q)= & \mp 287 k^{8}+1757 k^{7} q \mp 1470 k^{6} q^{2}-645 k^{5} q^{3} \pm 821 k^{4} q^{4} \\
& +278 k^{3} q^{5} \mp 278 k^{2} q^{6}-36 k q^{7} \mp 36 q^{8} .
\end{aligned}
$$

\footnotetext{
${ }^{10}$ Note that $|k-q|$ and $|k+q|$ do not contain $y$ and differ from $|\vec{k}-\vec{q}|=\left(k^{2}+q^{2}-2 q k y\right)^{1 / 2}$.
} 
Since Eqs. (4.30) and (4.31) are invariant for $k \rightarrow k \tau_{1}$ and $q \rightarrow q \tau_{1}$, we can rescale all the integrands through $\tau_{1}$ and expand the obtained results in powers of $|q-k| \tau_{1}$. The results for the derivatives of $\mathcal{F}_{\rho_{\mathrm{gw}}}^{(F P)}\left(x_{1}\right)$ and $\mathcal{F}_{\rho_{\mathrm{gw}}}^{(L L)}\left(x_{1}\right)$ with respect to $x_{1}$ will then be

$$
\begin{aligned}
\frac{d \mathcal{F}_{\rho_{\mathrm{gw}}}^{(F P)}}{d x_{1}}= & \frac{2624 \pi}{105 x_{1}}\left[1-\frac{2}{x_{1}}|q-k| \tau_{1}+\frac{3}{x_{1}^{2}}|q-k|^{2} \tau_{1}^{2}\right. \\
& \left.+\mathcal{O}\left(|q-k|^{3} \tau_{1}^{3}\right)\right] \\
\frac{d \mathcal{F}_{\rho_{\mathrm{gw}}}^{(L L)}}{d x_{1}}= & \frac{5632 \pi}{135|q-k| \tau_{1}}\left[1+\frac{419|q-k| \tau_{1}}{88 x_{1}}\right. \\
& \left.\quad-\frac{69|q-k|^{2}}{x_{1}^{2}}+\mathcal{O}\left(|q-k|^{3} \tau_{1}^{3}\right)\right] .
\end{aligned}
$$

By only keeping the leading terms in the expansions (4.33) and (4.34) the following pair of results shall be obtained:

$\mathcal{F}_{\rho_{\mathrm{gw}}}^{(F P)}(q) \simeq \frac{2624}{105} \pi \ln \left(\frac{\tau_{2}}{\tau_{1}}\right)$

$\mathcal{F}_{\rho_{\mathrm{gw}}}^{(L L)}(q) \simeq \frac{64}{135} \pi\left[331 \ln \left(\frac{\tau_{2}}{\tau_{1}}\right)-88 \ln \left(\frac{1-q \tau_{1}}{1-q \tau_{2}}\right)\right]$,

where the extrema of integration have been taken to be $k_{\max }=1 / \tau_{1}$ and $k_{\min }=1 / \tau_{2}$. It is natural to require that the largest wavelength (i.e. smallest wave number) is the one that will re-enter around the equality time since we are here considering wavelengths that exceed the Hubble radius during the radiation-dominated phase. In terms of a fiducial set of cosmological parameters, the following expression can be obtained:

$$
\ln \left(\frac{k_{\max }}{k_{\min }}\right)=58.77+\ln \mathcal{B}\left(r_{T}, \mathcal{A}_{\mathcal{R}}, \Omega_{\mathrm{M} 0}, \Omega_{\mathrm{R} 0}\right),
$$

where $^{11}$

$$
\begin{aligned}
\mathcal{B}\left(r_{T}, \mathcal{A}_{\mathcal{R}}, \Omega_{\mathrm{M} 0}, \Omega_{\mathrm{R} 0}\right)= & \left(\frac{r_{T}}{0.2}\right)^{1 / 4}\left(\frac{\mathcal{A}_{\mathcal{R}}}{2.41 \times 10^{-9}}\right)^{1 / 4} \\
& \times\left(\frac{h_{0}^{2} \Omega_{\mathrm{R} 0}}{4.15 \times 10^{-5}}\right)^{3 / 4}\left(\frac{h_{0}^{2} \Omega_{\mathrm{M} 0}}{0.1364}\right)^{-1}
\end{aligned}
$$

\footnotetext{
${ }^{11}$ We recall that $\Omega_{\mathrm{M} 0}$ and $\Omega_{\mathrm{R} 0}$ are the critical fractions of matter and radiation energy densities; $\mathcal{A}_{\mathcal{R}}$ is the amplitude of the conventional adiabatic mode and $r_{\mathrm{T}}$ is the ratio between the inflationary tensor spectrum and the spectrum of the adiabatic mode. The typical values correspond to the WMAP 9-year data [16] (see also Ref. [17]) supplemented by the Bicep2 data [18].
}

with the above choice of fiducial values we have that $\log \left(\tau_{2} / \tau_{1}\right) \simeq \mathcal{O}(60)$ in Eqs. (4.35)-(4.36).

The results reported so far demonstrate explicitly that the Landau-Lifshitz and Ford-Parker parametrizations are fully compatible insofar as they lead to equivalent results. Barring potential differences in the numerical prefactor we are led to the following expressions:

$$
\begin{aligned}
& \mathcal{P}_{\rho_{\mathrm{gw}}}^{(F P)}(q, \tau)=\mathcal{C}_{\rho_{\mathrm{gw}}}^{(F P)} \frac{H^{8}}{a^{4}(\tau)} q^{4} \tau_{1}^{4}, \\
& \mathcal{P}_{\rho_{\mathrm{gw}}}^{(L L)}(q, \tau)=\mathcal{C}_{\rho_{\mathrm{gw}}}^{(L L)} \frac{H^{8}}{a^{4}(\tau)} q^{4} \tau_{1}^{4},
\end{aligned}
$$

with $\mathcal{C}_{\rho_{\mathrm{gw}}}^{(F P)} \neq \mathcal{C}_{\rho_{\mathrm{gw}}}^{(L L)}$. This result is more than sufficient for the explicit solution of Eqs. (3.11) and (3.14). In what follows we shall therefore remove the superscripts specifying the parametrization of the energy-momentum pseudotensor and acknowledge that the net result of the whole calculation can be expressed as follows:

$$
\begin{aligned}
& \mathcal{P}_{\rho_{\mathrm{gw}}}(q, \tau)=\mathcal{C}_{\rho_{\mathrm{gw}}} H^{8}\left[\frac{a_{1}}{a(\tau)}\right]^{4} q^{4} \tau_{1}^{4}, \\
& \mathcal{P}_{p_{\mathrm{gw}}}(q, \tau)=\mathcal{C}_{p_{\mathrm{gw}}} H^{8}\left[\frac{a_{1}}{a(\tau)}\right]^{4} q^{4} \tau_{1}^{4}, \\
& \mathcal{P}_{\Pi_{\mathrm{gw}}}(q, \tau)=\mathcal{C}_{\Pi_{\mathrm{gw}}} H^{8}\left[\frac{a_{1}}{a(\tau)}\right]^{4} q^{4} \tau_{1}^{4} .
\end{aligned}
$$

The constants $\mathcal{C}_{\rho_{\mathrm{gw}}}, \mathcal{C}_{p_{\mathrm{gw}}}$ and $\mathcal{C}_{\Pi_{\mathrm{gw}}}$ do depend on the parametrization but they are of the same order. Equation (4.40) also contains the results for the pressure and the anisotropic stress that are derived by following exactly the same strategy detailed above for the case of the energy density. To avoid lengthy digressions the corresponding results for the pressure and the anisotropic stress have been collected in the Appendices (see, in particular, Appendix $\mathrm{B}$ for the pressure and Appendix $\mathrm{C}$ for the anisotropic stress). When $\tau>\tau_{2}$, Eq. (4.40) keeps in practice the same form (by replacing $\tau_{1}$ with $\tau_{2}$ and $a_{1}$ with $a_{2}$ ); the overall constants appearing in the new formulas will then be slightly different and the result will now hold in the limit

$$
\begin{aligned}
\tau & >\tau_{2}>\left|\tau_{1}\right|, \quad|q \tau| \ll 1, \\
\left|q \tau_{2}\right| & \ll 1, \quad|\vec{q}-\vec{k}| \tau_{2} \ll 1 .
\end{aligned}
$$

Let us finally consider the following pair of evolution equations obtained, respectively, in the Ford-Parker and in the Landau-Lifshitz parametrizations:

$$
\begin{aligned}
& \partial_{\tau} \delta \rho_{\mathrm{gw}}^{(F P)}+3 \mathcal{H}\left[\delta \rho_{\mathrm{gw}}^{(F P)}+\delta p_{\mathrm{gw}}^{(F P)}\right]-\vec{\nabla} \cdot \overrightarrow{\mathcal{J}}=0, \\
& \partial_{\tau} \delta \rho_{\mathrm{gw}}^{(L L)}+3 \mathcal{H}\left[\delta \rho_{\mathrm{gw}}^{(L L)}+\delta P_{\mathrm{gw}}^{(L L)}\right]-\vec{\nabla} \cdot \overrightarrow{\mathcal{J}}=0 .
\end{aligned}
$$


Equation (4.42) comes from Eq. (2.16) by requiring $\bar{\nabla}_{\mu} \mathcal{W}_{\nu}^{\mu}=0$, where $\bar{\nabla}_{\mu}$ denotes the covariant derivative defined in terms of the conformally flat metric $\bar{g}_{\mu \nu}$. Equation (4.43) is instead derived from the second-order (tensor) fluctuation of the Bianchi identity, i.e. $\delta_{\mathrm{t}}^{(2)}\left(\nabla_{\mu} \mathcal{G}_{\nu}^{\mu}\right)=0 . \quad$ The Bianchi identity itself (i.e. $\nabla_{\mu} \mathcal{G}_{\nu}^{\mu}=0$ ) is valid to all orders and it will be satisfied, in particular, to second order. As can be directly checked, Eqs. (4.42) and (4.43) are exactly equivalent to Eq. (2.10) once the corresponding definitions of the various fluctuations are taken into account.

The specific form of $\delta P_{\mathrm{gw}}^{(L L)}$ is given in Appendix B [see Eq. (B9)] and, unlike $\delta p_{\mathrm{gw}}^{(F P)}$, it contains an effective viscous contribution. Some authors $[19,20]$ suggested in the past that the relic gravitons may lead to an effective bulk viscosity in the early Universe. This is not exactly the viewpoint adopted here where the emphasis is on those results that are independent of the parametrization of the energy-momentum pseudotensor. In the long-wavelength limit, the last terms in Eqs. (4.42)-(4.43) are subleading in comparison with the other contributions of the corresponding equations. This occurrence is compatible with the form of the power spectra of Eqs. (4.40) and it implies, in the long-wavelength limit (and in spite of the parametrization) that $\delta p_{\mathrm{gw}} \simeq-\delta \rho_{\mathrm{gw}} / 3$ and that $\delta \rho_{\mathrm{gw}} \simeq a^{-2}$.

\section{The induced curvature perturbations}

Equations (4.40) and (4.42)-(4.43) are essential for the explicit evaluation of the integrals appearing in Eq. (3.14). The first line of Eq. (3.14) contains two factors: the adiabatic piece (which is left untouched by the present discussion) and a further contribution (depending on the first time derivative of $\mathcal{R}$ at $\tau_{1}$ ). This term decreases in time and it can be soon neglected for $\tau>\tau_{1}$. The latter conclusion follows by observing that $z_{\mathrm{t}}(\tau)$ is proportional to $a(\tau)$ : during radiation (i.e. $\left.\mathcal{H}^{\prime}=-\mathcal{H}^{2}\right) \quad z_{\mathrm{t}}^{2}(\tau)=$ $12 a^{2}(\tau) / \ell_{\mathrm{P}}^{2}$. This result implies that

$$
z_{\mathrm{t}}^{2}\left(\tau_{1}\right) \int_{\tau_{1}}^{\tau} \frac{d \tau^{\prime}}{z_{\mathrm{t}}^{2}\left(\tau^{\prime}\right)}=a^{2}\left(\tau_{1}\right) \mathcal{H}_{1}\left(1-\frac{\tau_{1}}{\tau}\right) .
$$

Moreover, in the purely adiabatic case, $\mathcal{R}^{\prime}\left(k, \tau_{1}\right)=0$ and therefore the prefactor multiplying Eq. (4.44) only depends on $\Sigma_{\mathcal{R}}\left(k, \tau_{1}\right)$.

Having discussed the first line of Eq. (3.14) we are left with the remaining terms that can be written as

$\mathcal{I}_{1}(\vec{q}, \tau)=\int_{\tau_{1}}^{\tau} \Sigma_{\mathcal{R}}\left(\vec{q}, \tau^{\prime}\right) d \tau^{\prime}$,

$\mathcal{I}_{2}(\vec{q}, \tau)=3 \int_{\tau_{1}}^{\tau} \frac{d \tau^{\prime}}{z_{\mathrm{t}}^{2}\left(\tau^{\prime}\right)} \int_{\tau_{1}}^{\tau^{\prime}} a^{4}\left(\tau^{\prime \prime}\right) \Pi_{\mathrm{gw}}\left(\vec{q}, \tau^{\prime \prime}\right)$.

Owing to the specific form of the power spectra of Eq. (4.40) and thanks to Eqs. (4.42)-(4.43) we can also write the Fourier-space components of the various fluctuations in the following factorized form:

$$
\begin{aligned}
\delta \rho_{\mathrm{gw}}(\vec{q}, \tau) & =\frac{\delta \rho_{\mathrm{gw}}(\vec{q})}{a^{2}(\tau)}, \\
\delta p_{\mathrm{gw}}(\vec{q}, \tau) & =\frac{\delta p_{\mathrm{gw}}(\vec{q})}{a^{2}(\tau)}, \\
\Pi_{\mathrm{gw}}(\vec{q}, \tau) & =\frac{\Pi_{\mathrm{gw}}(\vec{q})}{a^{2}(\tau)} .
\end{aligned}
$$

Thanks to Eq. (4.47) the integrals of Eqs. (4.45)-(4.46) can be performed in explicit terms and the result is

$$
\begin{aligned}
& \mathcal{I}_{1}(\vec{q}, a)=\frac{\mathcal{E}_{1}(\vec{q})}{H^{2} \bar{M}_{\mathrm{P}}^{2}} \mathcal{D}_{1}(w, a), \\
& \mathcal{I}_{2}(\vec{q}, a)=\frac{\mathcal{E}_{2}(\vec{q})}{H^{2} \bar{M}_{\mathrm{P}}^{2}} \mathcal{D}_{2}(w, a),
\end{aligned}
$$

where $\bar{M}_{\mathrm{P}}=\ell_{\mathrm{P}}^{-1}$ and $^{12}$

$$
\begin{aligned}
\mathcal{E}_{1}(\vec{q}) & =c_{\mathrm{st}}^{2} \delta \rho_{\mathrm{gw}}(\vec{q})-\delta p_{\mathrm{gw}}(\vec{q})+\Pi_{\mathrm{gw}}(\vec{q}), \\
\mathcal{E}_{2}(\vec{q}) & =\Pi_{\mathrm{gw}}(\vec{q}), \\
\mathcal{D}_{1}(w, a) & =\frac{1}{3(3 w+1)(w+1)}\left[\left(\frac{a}{a_{1}}\right)^{3 w+1}-1\right], \\
\mathcal{D}_{2}(w, a)= & \frac{w(3 w+1)}{6(w-1)(w+1)}\left[1+\frac{3(w-1)}{(3 w+5)}\left(\frac{a}{a_{1}}\right)^{3 w+1}\right. \\
& \left.-\frac{2(3 w+1)}{(3 w+5)}\left(\frac{a}{a_{1}}\right)^{3(w-1) / 2}\right] .
\end{aligned}
$$

The result for $\mathcal{D}_{2}(w, a)$ is correct provided $w \neq 1$; in the case $w=1$ (stiff post-inflationary phase) the results are physically equivalent. ${ }^{13}$

Using the results of Eqs. (4.45), (4.46) and (4.48) the power spectrum of curvature perturbations is given by

$\mathcal{P}_{\mathcal{R}}(q, \tau)=\mathcal{A}_{\mathcal{R}}+\left(\frac{H}{\bar{M}_{\mathrm{P}}}\right)^{4} q^{4} \tau_{1}^{4}\left[\mathcal{C}_{1} \mathcal{D}_{1}(w, a)+\mathcal{C}_{2} \mathcal{D}_{2}(w, a)\right]^{2}$,

$$
\mathcal{C}_{1}=c_{\mathrm{st}}^{2} \sqrt{\mathcal{C}_{\rho_{\mathrm{gw}}}}-\sqrt{\mathcal{C}_{p_{\mathrm{gw}}}}+\sqrt{\mathcal{C}_{\Pi_{\mathrm{gw}}}}, \quad \mathcal{C}_{2}=\sqrt{\mathcal{C}_{\Pi_{\mathrm{gw}}}} .
$$

\footnotetext{
${ }^{12}$ In principle the interesting cases are $w=1 / 3$ and $w=0$. However, the analysis reported in the preceding section can be generalized to the case of a generic post-inflationary phase with the result that Eqs. (4.40) and (4.42)-(4.43) are formally valid.

${ }^{13}$ In the case of a stiff post-inflationary phase $\mathcal{D}_{1}(1, a)=$ $\left[\left(a / a_{1}\right)^{2}-1\right] / 24$ and $\mathcal{D}_{2}(1, a)=\left[\left(a / a_{1}\right)^{4}-1-2 \ln \left(a / a_{1}\right)\right] / 4$. The analysis of the correlation functions can be conducted also in this case with results that are quantitatively compatible with the ones of the radiation case. This analysis will not be reported in detail since it is not central to the theme of this paper.
} 
Consider now two specific (but important) cases, namely $w=1 / 3$ (radiation-dominated case) and $w=0$ (matterdominated $\left.\operatorname{case}^{14}\right)$. In the radiation case, recalling that $H / \mathrm{M}_{\mathrm{P}}=\pi \sqrt{r_{T} \mathcal{A}_{\mathcal{R}} / 2}$, we have

$\mathcal{P}_{\mathcal{R}}(q, \tau) \simeq \mathcal{A}_{\mathcal{R}}+\frac{r_{T}^{2}}{576} \mathcal{A}_{\mathcal{R}}^{2} q^{4} \tau_{1}^{4}\left(3 \mathcal{C}_{1}+\mathcal{C}_{2}\right)^{2}\left(\frac{a}{a_{1}}\right)^{4}$.

The wavelength of the fluctuation becomes comparable with the Hubble radius at the re-entry time namely when $q \tau_{\text {re }}=1$. Let us suppose, as implied by Eq. (4.51) that the given mode re-enters during the radiation-dominated phase:

$\mathcal{P}_{\mathcal{R}}\left(q, \tau_{\mathrm{re}}\right) \simeq \mathcal{A}_{\mathcal{R}}+\frac{r_{T}^{2}}{576} \mathcal{A}_{\mathcal{R}}^{2}\left(3 \mathcal{C}_{1}+\mathcal{C}_{2}\right)^{2} q^{4} \tau_{1}^{4}\left(\frac{\tau_{\mathrm{re}}}{\tau_{1}}\right)^{4}$.

Since $q \tau_{1}\left(a_{\mathrm{re}} / a_{1}\right)=q \tau_{\mathrm{re}}$ (and, by definition, $q \tau_{\mathrm{re}}=1$ ) Eq. (4.52) also implies that

$$
\mathcal{P}_{\mathcal{R}}\left(q, \tau_{\mathrm{re}}\right) \simeq \mathcal{A}_{\mathcal{R}}+\frac{9 \mathcal{C}_{\rho_{\mathrm{gw}}}}{144} r_{T}^{2} \mathcal{A}_{\mathcal{R}}^{2}
$$

In Eq. (4.53) we used the following chain of equalities:

$$
\frac{1}{576}\left(3 \mathcal{C}_{1}+\mathcal{C}_{2}\right)^{2} \simeq \frac{1}{144}\left(\sqrt{\mathcal{C}_{\rho_{\mathrm{gw}}}}+2 \sqrt{\mathcal{C}_{\Pi_{\mathrm{gw}}}}\right)^{2} \simeq \frac{9 \mathcal{C}_{\rho_{\mathrm{gw}}}}{144} .
$$

The last equality on the right-hand side of Eq. (4.54) follows by assuming that $\mathcal{C}_{\Pi_{\mathrm{gw}}}$ and $\mathcal{C}_{\rho_{\mathrm{gw}}}$ are of the same order. On the basis of the estimates previously discussed within the different parametrizations, the whole numerical factor multiplying $r_{T}^{2} \mathcal{A}_{\mathcal{R}}^{2}$ in the second term on the righthand side of Eq. (4.53) can be estimated to be between $2 \times 10^{2}$ and $5 \times 10^{2}$. Finally, if the given mode re-enters during the matter-dominated phase we have

$$
\mathcal{P}_{\mathcal{R}}\left(q, \tau_{\mathrm{re}}\right) \simeq \mathcal{A}_{\mathcal{R}}+\frac{9 \overline{\mathcal{C}}_{\rho_{\mathrm{gw}}}}{144} r_{T}^{2} \mathcal{A}_{\mathcal{R}}^{2} q^{4} \tau_{1}^{4}\left(\frac{a_{\mathrm{eq}}}{a_{1}}\right)^{4}\left(\frac{a_{\mathrm{re}}}{a_{\mathrm{eq}}}\right)^{2},
$$

which gives back the result of Eq. (4.52) since, during the matter-dominated phase, we have that $\left(a_{\text {re }} / a_{\mathrm{eq}}\right)^{2} \simeq$ $\left(\tau_{\text {re }} / \tau_{\text {eq }}\right)^{4}$. In Eq. (4.55) we defined $\overline{\mathcal{C}}_{\rho_{\mathrm{gw}}}$ which is numerically different from $\mathcal{C}_{\rho_{\mathrm{gw}}}$ but it has the same origin and it is of the same order.

The rationale for the result of Eqs. (4.51) and (4.55) rests on the fact that the modification induced by the graviton fluid on the scalar power spectrum goes as $q^{4} \tau^{4}$ which is, at most, of order 1 at the moment when the mode re-enters the Hubble radius. Before re-entry $q \tau \ll 1$ and the correction

\footnotetext{
${ }^{14}$ Notice that if $w \rightarrow 0 \mathcal{D}_{2} \rightarrow 0$ but $\mathcal{D}_{1} \neq 0$.
}

induced by the gravitons is negligible. It is important to appreciate that this result does not conflict with quantummechanical calculations performed directly during the de Sitter stage of expansion [21]. The observation of Ref. [21] can be summarized by saying that at $n$th order in perturbation theory the correlation function of curvature perturbations during a quasi-de Sitter stage of expansion can grow, at most, as $(\ln a)^{n}$. This limitation in the growth holds to leading order in $q /(a H) \simeq q \tau$ i.e. up to terms that are small while the given modes are larger than the Hubble radius.

\section{CONCLUDING REMARKS}

The fluctuations of the energy density of the long- and short-wavelength gravitons are strongly suppressed relative to the background energy density which is roughly constant during the inflationary phase. The same statement holds for the fluctuations of the pressure and for the anisotropic stress. Conversely the same fluctuations induce curvature perturbations that grow during the post-inflationary epoch.

Barring some evolution in the number of relativistic species of the plasma, after the end of inflation the shortwavelength gravitons produce scalar inhomogeneities that decrease roughly at the same rate as the radiation background: if they were small at the end of inflation they will remain small. The fluctuations of the energy density, pressure and anisotropic stress of the long-wavelength gravitons decrease in time at a rate that it is slower than the one of the radiation and matter energy densities. In this situation the curvature perturbations inherit a supplementary contribution that depends on the ratio between the fluctuations of the energy-momentum pseudotensor of the relic gravitons and the background energy density.

The corrections to the power spectrum of the curvature perturbations can then be computed and they mildly depend on the parametrizations of the energy-momentum pseudotensor. Since the definition of the momentum and energy of the gravitational field itself is formally not unique, a more pragmatic approach has been adopted. The same analysis has been performed using completely different assignments of the energy-momentum pseudotensor. A posteriori, the compatibility of the obtained results fully justifies our strategy. It turns out that the growth of the curvature perturbations induced by this effect is approximately compensated by the corresponding spectral slope.

All in all, we argue that the fluctuations of the longwavelength gravitons can be treated in the fluid approximation but different pseudotensors suggest slightly different properties of the effective fluid. For instance the classic Landau-Lifshitz parametrization (appropriately generalized to the case of conformally flat backgrounds) suggests that the gravitons contribute to an effective bulk viscosity of the corresponding fluid. If we choose instead to parametrize the energy-momentum pseudotensor in terms of two minimally coupled scalar fields (one for each polarization of the graviton) the effective fluid is inviscid. While the 
optimal parametrization of the energy-momentum pseudotensor of the relic gravitons is not at issue, the present findings show, more modestly, that different strategies lead, in practice, to consistent physical results.

\section{ACKNOWLEDGEMENTS}

It is a pleasure to thank Jens Vigen and Tullio Basaglia of the CERN scientific information service for their valuable assistance.

\section{APPENDIX A: POWER SPECTRUM OF THE ENERGY DENSITY}

The power spectrum of the energy density in the LandauLifshitz parametrization has been discussed in the bulk of the paper but we report here, for the sake of precision, the explicit expressions of $\mathcal{A}_{1}(\vec{k}, \vec{q}, \tau), \mathcal{A}_{2}(\vec{k}, \vec{q}, \tau)$ and $\mathcal{A}_{3}(\vec{k}, \vec{q}, \tau)$ appearing in Eq. (4.6):

$$
\begin{aligned}
\mathcal{A}_{1}(\vec{k}, \vec{q}, \tau)= & \mathcal{P}_{f f}(k, \tau) \mathcal{P}_{g g}(|\vec{q}-\vec{k}|, \tau)+\mathcal{P}_{f f}(|\vec{q}-\vec{k}|, \tau) \mathcal{P}_{g g}(k, \tau) \\
& +\mathcal{P}_{f g}(k, \tau) \mathcal{P}_{g f}(|\vec{q}-\vec{k}|, \tau)+\mathcal{P}_{f g}(|\vec{q}-\vec{k}|, \tau) \mathcal{P}_{g f}(k, \tau), \\
\mathcal{A}_{2}(\vec{k}, \vec{q}, \tau)= & \mathcal{P}_{f g}(k, \tau) \mathcal{P}_{g g}(|\vec{q}-\vec{k}|, \tau)+\mathcal{P}_{f g}(|\vec{q}-\vec{k}|, \tau) \mathcal{P}_{g g}(k, \tau)+\mathcal{P}_{g f}(k, \tau) \mathcal{P}_{g g}(|\vec{q}-\vec{k}|, \tau)+\mathcal{P}_{g f}(|\vec{q}-\vec{k}|, \tau) \mathcal{P}_{g g}(k, \tau) \\
& +[\vec{k} \cdot(\vec{q}-\vec{k})]\left[\mathcal{P}_{f f}(k, \tau)\left(\mathcal{P}_{g f}(|\vec{q}-\vec{k}|, \tau)+\mathcal{P}_{f g}(|\vec{q}-\vec{k}|, \tau)\right)+\mathcal{P}_{f f}(|\vec{q}-\vec{k}|, \tau)\left(\mathcal{P}_{g f}(k, \tau)+\mathcal{P}_{f g}(k, \tau)\right)\right], \quad \\
\mathcal{A}_{3}(\vec{k}, \vec{q}, \tau)= & \mathcal{P}_{g g}(k, \tau) \mathcal{P}_{g g}(|\vec{q}-\vec{k}|, \tau)+[\vec{k} \cdot(\vec{q}-\vec{k})]^{2} \mathcal{P}_{f f}(k, \tau) \mathcal{P}_{f f}(|\vec{q}-\vec{k}|, \tau) \\
& +\vec{k} \cdot(\vec{q}-\vec{k})\left(\mathcal{P}_{f g}(k, \tau) \mathcal{P}_{f g}(|\vec{q}-\vec{k}|, \tau)+\mathcal{P}_{g f}(k, \tau) \mathcal{P}_{g f}(|\vec{q}-\vec{k}|, \tau)\right) .
\end{aligned}
$$

\section{APPENDIX B: POWER SPECTRUM OF THE PRESSURE}

The same strategy adopted for the calculation of the fluctuations of the energy density can be pursued to compute the fluctuations of the pressure. The discussion on the differences between the two parametrizations shall not be repeated here since it mirrors exactly the results mentioned in the case of the energy density. The fluctuations of the pressure and the corresponding two-point function are defined exactly as in the case of the energy density:

$$
\begin{aligned}
& \delta p_{\mathrm{gw}}(\vec{x}, \tau)=\frac{1}{(2 \pi)^{3 / 2}} \int d^{3} k e^{-i \vec{k} \cdot \vec{x}} \delta p_{\mathrm{gw}}(\vec{k}, \tau), \\
& \left\langle\delta p_{\mathrm{gw}}(\vec{p}, \tau) \delta p_{\mathrm{gw}}(\vec{q}, \tau)\right\rangle=\frac{2 \pi^{2}}{q^{3}} \delta^{(3)}(\vec{q}+\vec{p}) \mathcal{P}_{p_{\mathrm{gw}}}(q, \tau) .
\end{aligned}
$$

The fluctuations of the pressure in the two parametrizations are, respectively,

$$
\begin{aligned}
\delta p_{\mathrm{gw}}^{(F P)}(\vec{q}, \tau)= & \frac{1}{8 \ell_{\mathrm{P}}^{2} a^{2}} \int \frac{d^{3} k}{(2 \pi)^{3 / 2}}\left[\hat{H}_{\ell m}(\vec{k}, \tau) \hat{H}_{\ell m}(\vec{q}-\vec{k}, \tau)\right. \\
& \left.-\frac{(\vec{k}-\vec{q}) \cdot \vec{k}}{3} \hat{h}_{\ell m}(\vec{k}, \tau) \hat{h}_{\ell m}(\vec{q}-\vec{k}, \tau)\right], \quad(\mathrm{B} 3) \\
\delta p_{\mathrm{gw}}^{(L L)}(\vec{q}, \tau)= & -\frac{1}{24 \ell_{\mathrm{P}}^{2} a^{2}} \int \frac{d^{3} k}{(2 \pi)^{3 / 2}}\left[5 \hat{H}_{\ell m}(\vec{k}, \tau) \hat{H}_{\ell m}(\vec{q}-\vec{k}, \tau)\right. \\
& \left.-7[(\vec{k}-\vec{q}) \cdot \vec{k}] \hat{h}_{\ell m}(\vec{k}, \tau) \hat{h}_{\ell m}(\vec{q}-\vec{k}, \tau)\right] .
\end{aligned}
$$

We first analyze the correlation of the pressure in the Ford-Parker parametrization. In this case the approximate result for the power spectrum of the pressure is

$$
\begin{aligned}
\mathcal{P}_{p_{\mathrm{gw}}}^{(F P)}(q, \tau)= & \frac{q^{3}}{1152 \pi^{5} \ell_{\mathrm{P}}^{4} a^{4}} \int \frac{d^{3} k}{k^{3}|\vec{q}-\vec{k}|^{3}}[\vec{k} \cdot(\vec{k}-\vec{q})]^{2} \\
& \times Q(\vec{k}, \vec{q}) P_{f f}(k, \tau) P_{f f}(|\vec{q}-\vec{k}|, \tau) .
\end{aligned}
$$

The result obtained for the pressure can be expressed by saying that the power spectra of the energy density and of the pressure are related as

$$
\mathcal{P}_{p_{\mathrm{gw}}}^{(F P)}(q, \tau) \simeq \frac{\mathcal{P}_{\rho_{\mathrm{gw}}}^{(F P)}(q, \tau)}{9} .
$$

This result can be understood on the basis of Eq. (4.42):

$$
\partial_{\tau} \delta \rho_{\mathrm{gw}}^{(F P)}+3 \mathcal{H}\left[\delta \rho_{\mathrm{gw}}^{(F P)}+\delta p_{\mathrm{gw}}^{(F P)}\right]-\vec{\nabla} \cdot \overrightarrow{\mathcal{J}}=0 .
$$

The third term in Eq. (B7) is subleading in comparison with the other two when the relevant wavelengths exceed the Hubble radius at the corresponding epoch. Now, the evolution of the power spectrum of the energy density deduced in Sec. IV implies that $\delta \rho_{\mathrm{gw}}^{(F P)}(\vec{q}, \tau)$ scales as $a^{-2}$ which is compatible with Eq. (B7) provided the effective barotropic index when the relevant wavelengths exceed the Hubble radius is $\mathcal{O}(-1 / 3)$. This result is in turn compatible with Eq. (B6). 
As we saw in Sec. IV the different parametrizations are physically equivalent and differ, at the level of the power spectrum, just by the specific value of the overall constant. If this is true we expect that the same result of Eq. (B7) can also be obtained in the framework of the Landau-Lifshitz parametrization where the evolution of the energy density and pressure fluctuation comes by perturbing to second order the Bianchi identity that must hold at any order in the perturbative expansion. The result of this procedure implies that the equation obeyed is ${ }^{15}$

$$
\partial_{\tau} \delta \rho_{\mathrm{gw}}^{(L L)}+3 \mathcal{H}\left[\delta \rho_{\mathrm{gw}}^{(L L)}+\delta P_{\mathrm{gw}}^{(L L)}\right]-\vec{\nabla} \cdot \overrightarrow{\mathcal{J}}=0,
$$

where $\delta P_{\mathrm{gw}}^{(L L)}$ does not coincide with $\delta p_{\mathrm{gw}}^{(L L)}$ given in Eq. (B4) but it is defined as

$$
\delta P_{\mathrm{gw}}^{(L L)}=\delta p_{\mathrm{gw}}^{(L L)}+\frac{\mathcal{H}^{2}-\mathcal{H}^{\prime}}{3 \mathcal{H} a^{2} \ell_{\mathrm{P}}^{2}} H_{\ell m} h_{\ell m}
$$

We can therefore compute the power spectra of $\delta p_{\mathrm{gw}}^{(L L)}$ and of $\delta P_{\mathrm{gw}}^{(L L)}$. Defining $\mathcal{P}_{p_{\mathrm{gw}}}^{(L L)}(q, \tau)$ as the power spectrum of $\delta p_{\mathrm{gw}}^{(L L)}$ we have

$$
\mathcal{P}_{p_{\mathrm{gw}}}^{(L L)}(q, \tau) \simeq \frac{49}{25} \mathcal{P}_{\rho_{\mathrm{gw}}}^{(L L)}(q, \tau)
$$

This result can be obtained by using exactly the same techniques described in the previous section. However, defining $\overline{\mathcal{P}}_{P_{\mathrm{gw}}}^{(L L)}(q, \tau)$ as the power spectrum of $\delta P_{\mathrm{gw}}^{(L L)}$ we have

$$
\overline{\mathcal{P}}_{P_{\mathrm{gw}}}^{(L L)}(q, \tau) \simeq \frac{1}{3} \mathcal{P}_{\rho_{\mathrm{gw}}}^{(L L)}(q, \tau) .
$$

The dictionary between the two parametrizations of the energy-momentum pseudotensor implies therefore that $\delta p_{\mathrm{gw}}^{(F P)}$ is directly comparable with $\delta P_{\mathrm{gw}}^{(L L)}$ (rather than with $\left.\delta p_{\mathrm{gw}}^{(L L)}\right)$.

\section{APPENDIX C: POWER SPECTRUM OF THE ANISOTROPIC STRESS}

In spite of the specific parametrization of the anisotropic stress we shall have that

$$
\Pi_{\mathrm{gw}}(\vec{q}, \tau)=\frac{q_{i} q_{j}}{q^{2}} \Pi_{i j}(\vec{q}, \tau)
$$

The fluctuations of the energy density in the Ford-Parker and Landau-Lifshitz parametrizations are, respectively

$$
\begin{aligned}
\Pi_{\mathrm{gw}}^{(F P)}(\vec{q}, \tau)= & \frac{1}{12 \ell_{\mathrm{P}}^{2} a^{2}} \int \frac{d^{3} k}{(2 \pi)^{3 / 2}}\left[2(\vec{q} \cdot \vec{k})-3 \frac{(\vec{q} \cdot \vec{k})^{2}}{q^{2}}+k^{2}\right] \hat{h}_{\ell m}(\vec{k}, \tau) \hat{h}_{\ell m}(\vec{q}-\vec{k}, \tau), \\
\Pi_{\mathrm{gw}}^{(L L)}(\vec{q}, \tau)= & \frac{1}{12 \ell_{\mathrm{P}}^{2} a^{2}} \int \frac{d^{3} k}{(2 \pi)^{3 / 2}}\left\{2 \hat{H}_{\ell m}(\vec{k}, \tau) \hat{H}_{\ell m}(\vec{q}-\vec{k}, \tau)+\left[\vec{k} \cdot(\vec{q}-\vec{k})+3(\vec{k} \cdot \vec{q})-3 \frac{(\vec{k} \cdot \vec{q})^{2}}{q^{2}}\right] \hat{h}_{\ell m}(\vec{k}, \tau) \hat{h}_{\ell m}(\vec{q}-\vec{k}, \tau)\right. \\
& \left.-6 \frac{q^{i} q^{j}}{q^{2}}\left[\vec{k} \cdot(\vec{q}-\vec{k}) \hat{h}_{m i}(\vec{k}, \tau) \hat{h}_{m j}(\vec{q}-\vec{k}, \tau)-\hat{h}_{m i}(\vec{k}, \tau) \hat{h}_{m j}(\vec{q}-\vec{k}, \tau)\right]\right\} .
\end{aligned}
$$

The power spectrum of the anisotropic stress will only be computed in the Ford-Parker parametrization. As in the cases of the energy density and of the pressure we have that

$$
\begin{gathered}
\mathcal{P}_{\Pi}(q, \tau)=\frac{\left|q \tau_{1}\right|^{4}}{1152 \pi^{5}} \frac{H^{8}}{a^{4}} \mathcal{F}_{\Pi}^{(F P)}(q), \\
\mathcal{F}_{\Pi}^{(F P)}(q)=\int d^{3} k \frac{\left[2(\vec{q} \cdot \vec{k}) q^{2}-3(\vec{q} \cdot \vec{k})^{2}+k^{2} q^{2}\right]^{2}}{q^{5} k^{7}|\vec{q}-\vec{k}|^{7}}\left[k^{2}|\vec{q}-\vec{k}|^{2}+3\left(\vec{k} \cdot q-k^{2}\right)^{2}\right]\left[k^{2}|\vec{q}-\vec{k}|^{2}+\left(\vec{k} \cdot q-k^{2}\right)^{2}\right] .
\end{gathered}
$$

Using the techniques already employed in Sec. IV we can get

\footnotetext{
${ }^{15}$ It should be clear that $\delta P_{\mathrm{gw}}^{(L L)}$ differs from $\mathcal{P}_{\mathrm{gw}}^{(L L)}$ and $\overline{\mathcal{P}}_{\mathrm{gw}}^{(L L)}$. While $\delta P_{\mathrm{gw}}^{(L L)}$ denotes the pressure fluctuation in the Landau-Lifshitz parametrization [and in the presence of a viscous contribution, see Eq. (B9)], $\mathcal{P}_{p_{\mathrm{gw}}}^{(L L)}$ and $\overline{\mathcal{P}}_{P_{\mathrm{gw}}}^{(L L)}$ are the power spectra defined, respectively, from $\delta p_{\mathrm{gw}}^{(L L)}$ and $\delta P_{\mathrm{gw}}^{(L L)}$. With this caveat any potential clash of notation is avoided.
} 


$$
\mathcal{F}_{\Pi}^{(F P)}(q)=\frac{13504 \pi}{1155} \ln \left(\frac{\tau_{2}}{\tau_{1}}\right) .
$$

These results show indeed that $\mathcal{C}_{\rho_{\mathrm{gw}}}, \mathcal{C}_{p_{\mathrm{gw}}}$ and $\mathcal{C}_{\Pi_{\mathrm{gw}}}$ are all of the same order, as discussed in Sec. IV. Similar results hold in the Landau-Lifshitz parametrization.

[1] L. P. Grishchuk, Zh. Éksp. Teor. Fiz. 67, 825 (1974) [Sov. Phys. JETP 40, 409 (1975)]; Ann. N.Y. Acad. Sci. 302, 439 (1977).

[2] A. A. Starobinsky, JETP Lett. 30, 682 (1979); V. A. Rubakov, M. V. Sazhin, and A. V. Veryaskin, Phys. Lett. B 115, 189 (1982).

[3] R. Fabbri and M. D. Pollock, Phys. Lett. B 125, 445 (1983); L. F. Abbott and M. B. Wise, Nucl. Phys. 244, 541 (1984).

[4] L. P. Grishchuk and M. Solokhin, Phys. Rev. D 43, 2566 (1991); V. Sahni, Phys. Rev. D 42, 453 (1990).

[5] M. Giovannini, Phys. Rev. D 58, 083504 (1998); 60, 123511 (1999); Classical Quantum Gravity 26, 045004 (2009); W. Zhao, D. Baskaran, and P. Coles, Phys. Lett. B 680, 411 (2009).

[6] M. S. Pshirkov and D. Baskaran, Phys. Rev. D 80, 042002 (2009); T. Chiba, K. Kamada, and M. Yamaguchi, Phys. Rev. D 81, 083503 (2010); M. W. Hossain, R. Myrzakulov, M. Sami, and E. N. Saridakis, Phys. Rev. D 89, 123513 (2014).

[7] C. W. Misner, K. S. Thorne, and J. A. Wheeler, Gravitation (Freeman, New York, 1973), p. 467.

[8] S. Weinberg, Gravitation and Cosmology (Wiley, New York, 1972), p. 166.

[9] L. D. Landau and E. M. Lifshitz, The Classical Theory of Fields (Pergamon Press, New York, 1971).

[10] R. Isaacson, Phys. Rev. 166, 1263 (1968); 166, 1272 (1968).

[11] L. R. Abramo, R. Brandenberger, and V. Mukahanov, Phys. Rev. D 56, 3248 (1997); L. R. Abramo, Phys Rev. D 60, 064004 (1999); S. V. Babak and L. P. Grishchuk, Phys. Rev. D 61, 024038 (1999).

[12] M. Giovannini, Phys. Rev. D 73, 083505 (2006); D. Su and Y. Zhang, Phys. Rev. D 85, 104012 (2012).
[13] L. H. Ford and L. Parker, Phys. Rev. D 16, 1601 (1977); 16, 245 (1977); B. L. Hu and L. Parker, Phys. Lett A 63, 217 (1977).

[14] J. Bardeen, Phys. Rev. D 22, 1882 (1980); G. V. Chibisov and V. F. Mukhanov, Mon. Not. R. Astron. Soc. 200, 535 (1982); J. Bardeen, P. Steinhardt, and M. Turner, Phys. Rev. D 28, 679 (1983); J. A. Frieman and M. S. Turner, Phys. Rev. D 30, 265 (1984); H. Kodama and M. Sasaki, Prog. Theor. Phys. Suppl. 78, 1 (1984); J-c. Hwang, Astrophys. J. 375, 443 (1991).

[15] V. N. Lukash, Zh. Eksp. Teor. Fiz. 79, 1601 (1980) [ Sov. Phys. JETP 52, 807 (1980)]; V. Strokov, Astronomy Reports 51, 431 (2007).

[16] B. Gold et al., Astrophys. J. Suppl. Ser. 192, 15 (2011); D. Larson et al., Astrophys. J. Suppl. Ser. 192, 16 (2011); C. L. Bennett et al., Astrophys. J. Suppl. Ser. 192, 17 (2011); G. Hinshaw et al., Astrophys. J. Suppl. Ser. 20819 (2013); C. L. Bennett et al., Astrophys. J. Suppl. Ser. 20820 (2013).

[17] D. N. Spergel et al., Astrophys. J. Suppl. Ser. 148, 175 (2003); 170, 377 (2007); L. Page et al., Astrophys. J. Suppl. Ser. 170, 335 (2007).

[18] P. A. R. Ade et al. (BICEP2 Collaboration), Phys. Rev. Lett. 112, 241101 (2014); Astrophys. J. 792, 62 (2014).

[19] G. L. Murphy, Phys. Rev. D 8, 4231 (1973); Phys. Lett. A 62, 75 (1977).

[20] V. A. Belinskii and I. M. Khalatnikov, Zh. Eksp. Teor. Fiz. 69, 401 (1975) [ Sov. Phys. JETP 42, 205 (1976)]; Zh. Eksp. Teor. Fiz. Pis. Red. 21, 223 (1975) [JETP Lett. 21, 99 (1975)].

[21] S. Weinberg, Phys. Rev. D 72043514 (2005); 74, 023508 (2006). 